\title{
Dynamical friction in a gas: The supersonic case ${ }^{\star}$
}

\author{
Aaron T. Lee and Steven W. Stahler
}

\author{
Astronomy Department, University of California, Berkeley CA 94720, USA \\ e-mail: sstahler@astro.berkeley.edu
}

Received 10 October 2013 / Accepted 8 November 2013

\begin{abstract}
Any gravitating mass traversing a relatively sparse gas experiences a retarding force created by its disturbance of the surrounding medium. In a previous contribution, we determined this dynamical friction force when the object's velocity was subsonic. We now extend our analysis to the supersonic regime. As before, we consider small perturbations created in the gas far from the gravitating object, and thereby obtain the net influx of linear momentum over a large, bounding surface. Various terms in the perturbation series formally diverge, necessitating an approximate treatment of the flow streamlines. Nevertheless, we are able to derive exactly the force itself. As in the subsonic case, we find that $F=\dot{M} V$, where $\dot{M}$ is the rate of mass accretion onto the object and $V$ its instantaneous velocity with respect to distant background gas. Our force law holds even when the object is porous (e.g., a galaxy) or is actually expelling mass in a wind. Quantitatively, the force in the supersonic regime is less than that derived analytically by previous researchers, and is also less than was found in numerical simulations through the mid-1990s. We urge simulators to revisit the problem using modern numerical techniques. Assuming our result to be correct, it is applicable to many fields of astrophysics, ranging from exoplanet studies to galactic dynamics.
\end{abstract}

Key words. hydrodynamics - waves - ISM: kinematics and dynamics - planet-disk interactions - galaxies: kinematics and dynamics

\section{Introduction}

Whenever a massive object passes through a rarefied medium, it draws surrounding matter toward it. As a result, this material creates an overdense wake behind the object that exerts its own gravitational pull, retarding the original motion. Such dynamical friction arises whether the medium consists of non-interacting point particles, e.g., a stellar cluster, or a continuum fluid, e.g., an interstellar cloud. Chandrasekhar (1943) provided the essential theory when the background is collisionless, and his solution has been extensively used in studies of both star clusters and galaxies. Interaction of a gravitating object with gas also occurs in a wide variety of situations. A partial list of topics and references includes: the interaction of planets and gaseous disks (Teyssandier et al. 2013); the orbital decay of common-envelope binaries (Ricker \& Taam 2008); the settling of massive stars in dense molecular clouds (Chavarría et al. 2010); the coalescence of massive black holes in both isolated galactic nuclei (Narayan 2000) and colliding galaxies (Armitage \& Natarajan 2005); and the heating of intracluster gas by infalling galaxies (El-Zant et al. 2004).

Despite the widespread occurrence of gaseous dynamical friction, there is still no generally accepted derivation of the force, even after 70 years of effort. The flow in the vicinity of the gravitating mass is complex both temporally and spatially, as many simulations have shown (see, e.g. Matsuda et al. 1987). Theorists seeking a fully analytic expression for the timeaveraged force have employed various strategems to circumvent a detailed description of this region. In the course of their classic

* Appendix $\mathrm{A}$ is available in electronic form at http: //WwW . aanda.org studies of stellar accretion, Bondi \& Hoyle (1944) took the star to be traversing a zero-temperature gas. In their model, fluid elements follow hyperbolic orbits in the star's reference frame and land in an infinitely thin, dense spindle behind the object. Bondi \& Hoyle analyzed the transfer of linear momentum from the spindle to the star, and hence obtained the force. Dodd \& McCrae (1952) similarly investigated this hypersonic limit, as did, much more recently, Cantó et al. (2011). Dokuchaev (1964) first treated a finite-temperature gas. He determined the force by integrating the total power emitted by the object in acoustic waves (see also Rephaeli \& Salpeter 1980). Ruderman \& Spiegel (1971) used an impulse approximation in the reference frame of the background gas. Finally, Ostriker (1999) calculated the force by integrating directly over the wake, whose density she obtained through a linear perturbation analysis.

These researchers focused principally on the supersonic case, which is often the most interesting one astrophysically. That is, they took $V>c_{\mathrm{s}}$, where $V$ is the speed of the gravitating mass relative to the distant background, and $c_{\mathrm{S}}$ the sound speed in that gas. (In the hypersonic calculations of Bondi, Hoyle, and their successors, $c_{\mathrm{S}}$ was implicitly set to zero.) While their answers differed in detail, all agreed that the friction force in this regime varies as $V^{-2}$, with a coefficient that includes a Coulomb logarithm. This latter term also appears in the force derived by Chandrasekhar (1943), and arises from an integration in radius away from the mass. In all derivations, at least one of the integration limits is rather ill-defined.

The previous studies made two important, simplifying assumptions. First, they neglected any accretion of background gas by the moving object. Quantitatively, the assumption was that $R \gg r_{\text {acc }}$, where $R$ is the object's physical radius and 


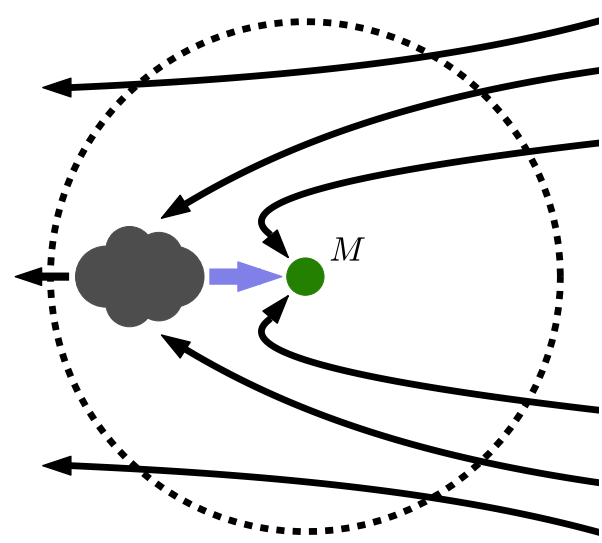

Fig. 1. Sketch of mass and momentum flow. Surrounding the central gravitating body of mass $M$ is a large, imaginary sphere. The thin curves represent streamlines of background gas. After entering the sphere, most gas simply exits again, while a small portion joins directly onto the mass. In addition, some gas temporarily forms an overdense wake, also sketched here. The wake tugs gravitationally on the mass, thereby imparting momentum to it (broad arrow). All gas entering the wake also leaves it, either joining the mass or exiting the sphere.

the accretion radius $r_{\text {acc }} \equiv 2 G M / V^{2}$ is the distance from the mass $M$ within which its gravity qualitatively alters the background flow. They further ignored $R$ compared to the scale for spatial variations in the surrounding flow. If $L$ denotes the latter scale, then these assumptions may be summarized as $L \gg R \gg r_{\text {acc }}$. Unfortunately, the inequality $R \gg r_{\text {acc }}$ is often not satisfied. For example, a low-mass star moving through a clusterproducing molecular cloud has $R \sim 10^{11} \mathrm{~cm}$ and $r_{\mathrm{acc}} \sim 10^{16} \mathrm{~cm}$. An analysis that covers this regime should treat the object as being point-like in all respects, allowing the possibility of mass accretion through infall.

This infall cannot occur via direct impact, since the geometrical cross section of the body is negligible by assumption. Instead, some fluid elements that initially miss the object are pulled back into it. Mass accretion is, in fact, closely related to dynamical friction. In the reference frame attached to the mass, the background gas flows by with a speed that approaches $V$ far away. The steady-state accretion rate onto the object is simply the net influx of mass through any closed surface surrounding it. Similarly, the friction force is the net influx of linear momentum. Much closer to the mass, this momentum influx manifests itself as two distinct force components. One is the gravitational pull from the wake, as described earlier. A second component is the direct advection of linear momentum from any background gas that falls into the object. Any determination of the force by an asymptotic surface integration cannot tease apart these two contributions.

To clarify these ideas, Fig. 1 shows graphically the mass and momentum flow within the extended gas cloud surrounding the central, gravitating object. Gas enters the region via the dotted sphere shown in the sketch. This gas then follows one of three paths. Most of it leaves the sphere downstream of the gravitating mass, as shown by the two outermost streamlines (thin solid curves). Only a small fraction of the gas makes its way to the deep interior. Some of it accretes onto the mass, first missing it and then looping back. Other gas that misses more widely is temporarily slowed by the gravitational pull of the mass and forms an overdense wake, also sketched in the figure. In steady state, the wake cannot gain net mass, so all of the gas entering it also leaves, either by joining onto the mass or exiting the sphere directly. Meanwhile, the wake itself tugs on the mass gravitationally. The broad arrow in the figure depicts this second form of momentum input to the mass. It is possible to determine the force analytically by integrating the net momentum flux over the bounding sphere. Numerical simulations can obtain this same force by calculating the advective and gravitational components acting directly on the mass.

In a previous paper (Lee \& Stahler 2011, hereafter Paper I), we did the analytic surface integration. We focused on the subsonic case, $V<c_{\mathrm{s}}$, which traditionally has been less explored ${ }^{1}$. Working in the reference frame whose origin is attached to the mass, we developed a perturbative method to analyze the small deviations of the background gas from a uniform, constantdensity, flow. By integrating the perturbed variables over a large sphere to obtain the net momentum influx, we arrived at a surprisingly simple result for the force: $F=\dot{M} V$. Here, $\dot{M}$ is the mass accretion rate onto the moving object. To evaluate this quantity from first principles, one would have to follow the trajectories of fluid elements as they accrete onto the central mass, and ensure that, following turnaround, they smoothly cross the sonic transition. This transition occurs well inside the region of validity for our calculation. Lacking a fundamental theory for $\dot{M}$, we adopted a variant of the interpolation formula of Bondi (1952) and thus found the force explicitly as a function of velocity. In this subsonic regime, our expression agrees reasonably well with past simulations (e.g., Ruffert 1996).

Here we apply the same technique to study the supersonic case. We quickly encounter the technical difficulty that some perturbation variables diverge, when expressed as functions of the angle from the background velocity vector. The true flow around the gravitating mass is, of course, well-defined everywhere, and the divergences simply indicate that the adopted series expansions fail at certain locations. Despite this mathematical inconvenience, we are able once again to derive the dynamical friction force through spatial integration of the linear momentum influx. The force has exactly the same form as previously: $F=\dot{M} V$. In the high-speed limit, we recover the $V^{-2}$ behavior found by others, but not the Coulomb logarithm.

In Sect. 2 below, we describe our solution strategy, and then formulate the problem using a convenient, non-dimensional scheme. Whenever material repeats that of Paper I, we abbreviate its presentation as much as possible. Section 3 analyzes the perturbed flow to first order only. In this approximation, we find there is no mass accretion or friction force, just as in the subsonic case. We extend the analysis to second order in Sect. 4, thus accounting for mass accretion. Here we also describe our method for calculating the flow numerically, and show sample results. Section 5 presents the analytic derivation of the force itself, and compares our expression to past simulations. Those found a greater force than we derive in the supersonic regime; we indicate possible causes for this discrepancy. Assuming our result to be correct, we present several representative applications. Finally, Sect. 6 compares our derivation to previous ones, and indicates directions for future work.

\section{Method of solution}

\subsection{Physical assumptions}

In the reference frame whose origin coincides with the mass, the background gas has speed $V$ far from the object and a spatially

1 Rephaeli \& Salpeter (1980) argued, based on a linear perturbation analysis, that the subsonic force vanishes. We showed in Paper I that extension of their analysis into the non-linear regime gives a finite result. 


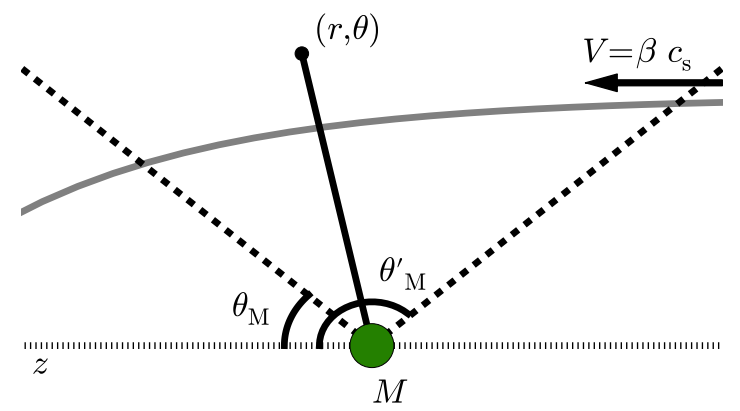

Fig. 2. Mathematical treatment of the flow. We erect a spherical coordinate system centered on the gravitating body. The gas is isothermal, and its velocity far upstream is $\beta c_{\mathrm{s}}(\beta>1)$. Indicated are the Mach angle $\theta_{M}$ and its supplement, $\theta_{M}^{\prime} \equiv \pi-\theta_{M}$.

uniform density, which we denote as $\rho_{0}$. We take the gas to be isothermal, with associated sound speed $c_{\mathrm{s}}$. As depicted in Fig. 2, we will be working in a spherical coordinate system $(r, \theta)$, and will assume that the gas flow is axisymmetric about the polar $(z-)$ axis, which is parallel to the asymptotic fluid velocity.

We neglect the self-gravity of the gas, and will be analyzing perturbations to the flow relatively far from the central mass. Specifically, our expansions are valid for $r \gg r_{\mathrm{s}}$, where $r_{\mathrm{s}} \equiv G M / c_{\mathrm{s}}^{2}$ is the sonic radius. We assume that in the far-field region of interest, the flow is steady-state. Of course, a steady flow cannot be established over arbitrarily large distances, and one must judge, in each astrophysical situation, whether the assumption is justified. In numerical simulations, which we later discuss by way of comparison, the flow occurs within some fixed computational volume, at the center of which lies the mass. For the non-relativistic case of relevance, the force of gravity propagates instantaneously, but alterations in the flow density and velocity take time. In practice, a steady flow is indeed approached in many such experiments (e.g., Pogorelov et al. 2000). The observed decay of transients is instructive, but again is only broadly suggestive of what may occur in Nature.

Figure 2 singles out two special angles, both of which only occur in supersonic flow. The first is the familiar Mach angle, defined through the relation

$\sin \theta_{M} \equiv 1 / \beta$

where $\beta \equiv V / c_{\mathrm{s}}$. The second is the supplement of the first: $\theta_{M}^{\prime} \equiv$ $\pi-\theta_{M}$. The figure of revolution swept out by the radius lying along $\theta=\theta_{M}$ is the Mach cone, while we dub the analogous figure swept out by $\theta=\theta_{M}^{\prime}$ the "anti-Mach cone". As Fig. 3 illustrates, we denote as the "upstream" region that portion of the flow bounded by the anti-Mach cone, while the "downstream" region lies within the Mach cone. Finally, the "intermediate" region has $\theta_{M}<\theta<\theta_{M}^{\prime}$.

A significant feature of the flow surrounding a gravitating mass is the presence of an accretion bowshock. Any fluid element that joins onto the mass penetrates to $r \lesssim r_{\text {acc }}$, and encounters a shock at $\theta \sim \theta_{M}$. At much larger $r$, such bowshocks weaken and degenerate to acoustic pulses, before fading away entirely (Zel'dovich \& Raizer 1968, Chap. 1). No such shock arises near $\theta_{M}^{\prime}$, even at relatively small distances. In summary, for $r \gg r_{\mathrm{s}} \gtrsim r_{\mathrm{acc}}$, we do not expect any discontinuities at $\theta_{M}$ or $\theta_{M}^{\prime}$ in steady-state flow, either in the fluid variables themselves or their derivatives. We need to keep this key point in mind as we encounter apparent discontinuities at both angles.

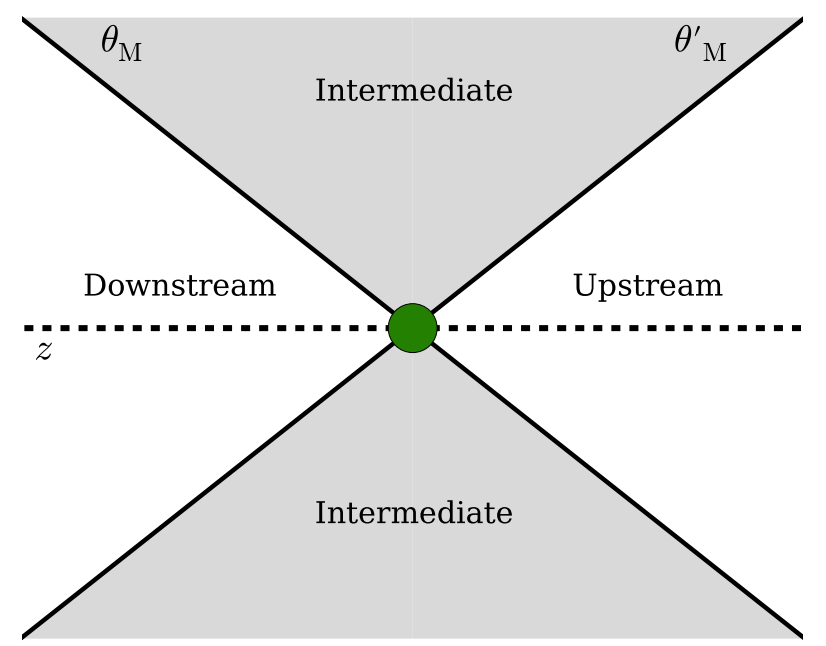

Fig. 3. Regions of the flow. The upstream and downstream regions lie within the anti-Mach and Mach cones, respectively. Between the two cones is the intermediate region. In the far-field flow, there are no physical barriers between the intermediate region and its neighbors, although mathematical divergences appear at the two cones.

\subsection{Mathematical formulation}

As in Paper I, we describe the flow through two dependent variables, the mass density $\rho(r, \theta)$ and the stream function $\psi(r, \theta)$. Individual velocity components may be recovered from these through the relations

$$
\begin{aligned}
& u_{r}=\frac{1}{\rho r^{2} \sin \theta} \frac{\partial \psi}{\partial \theta} \\
& u_{\theta}=\frac{-1}{\rho r \sin \theta} \frac{\partial \psi}{\partial r}
\end{aligned}
$$

which automatically ensure mass continuity. In the extreme farfield limit, the stream function approaches that for the background, uniform flow:

$$
\lim _{r \rightarrow \infty} \psi=\frac{\rho_{0} V r^{2} \sin ^{2} \theta}{2}=\rho_{0} c_{\mathrm{s}} r_{\mathrm{s}}^{2} \frac{\beta \sin ^{2} \theta}{2}\left(\frac{r}{r_{\mathrm{s}}}\right)^{2} .
$$

Our second form of the stream function's limit suggests how to expand $\psi(r, \theta)$ in a perturbation series valid for finite, but large, $r$ :

$\psi=\rho_{0} c_{\mathrm{s}} r_{\mathrm{s}}^{2}\left[f_{2}\left(\frac{r}{r_{\mathrm{s}}}\right)^{2}+f_{1}\left(\frac{r}{r_{\mathrm{s}}}\right)+f_{0}+f_{-1}\left(\frac{r}{r_{\mathrm{s}}}\right)^{-1}+\ldots\right]$.

Here, $f_{2} \equiv \beta \sin ^{2} \theta / 2$, while $f_{1}, f_{0}, f_{-1}$, etc., are as yet unknown, non-dimensional functions of $\beta$ and $\theta$. We write a similar perturbation expansion for the density:

$\rho=\rho_{0}\left[1+g_{-1}\left(\frac{r}{r_{\mathrm{s}}}\right)^{-1}+g_{-2}\left(\frac{r}{r_{\mathrm{s}}}\right)^{-2}+g_{-3}\left(\frac{r}{r_{\mathrm{s}}}\right)^{-3}+\ldots\right]$.

The quantities $g_{-1}, g_{-2}$, and $g_{-3}$ are again non-dimensional functions of $\beta$ and $\theta$, all of them unknown at this stage ${ }^{2}$.

2 The perturbation series in Eqs. (4) and (5) are valid for $r \gg r_{\mathrm{s}}$. On the other hand, we expect that a smooth flow, representing a modest perturbation of the background stream, is present beyond $r_{\text {acc }}$, which is much less than $r_{\mathrm{s}}$ in the hypersonic regime. We also expect our final expression for the force to be valid as long as the background gas extends beyond $r_{\text {acc }}$. 
The dynamical equations will be simplified once we recast all variables into non-dimensional form. We let the fiducial radius, density, and speed be $r_{\mathrm{s}}, \rho_{0}$, and $c_{\mathrm{s}}$, respectively, while we normalize the stream function to $\rho_{0} c_{\mathrm{s}} r_{\mathrm{s}}^{2}$. We will not change notation, but alert the reader whenever we revert to dimensional varables. Our fully non-dimensional perturbation series are

$\psi=f_{2} r^{2}+f_{1} r+f_{0}+f_{-1} r^{-1}+\ldots$

$\rho=1+g_{-1} r^{-1}+g_{-2} r^{-2}+g_{-3} r^{-3}+\ldots$

The task will be to solve for the various functions $f_{i}(\theta)$ and $g_{i}(\theta)$ appearing in these two series. (Henceforth, we will suppress the $\beta$-dependence for simplicity.) As explained in Sect. 2.3 of Paper I, the appropriate boundary conditions are $f_{i}(\pi)=f_{i}^{\prime}(\pi)=f_{i}^{\prime}(0)=0$ for $i=1,0,-1,-2$, etc., and $f_{i}(0)=0$ for $i=1,-1,-2$, etc. These conditions ensure regularity of both $u_{r}$ and $u_{\theta}$, as given by Eqs. (2) and (3), respectively, on both the upstream and downstream axes. Since $\sin \theta \rightarrow 0$ on both axes, the $r$ - and $\theta$-derivatives of $\psi$ must also vanish. Using the expansion of $\psi$ in Eq. (6), we derive the aforementioned conditions. There is no associated restriction on $f_{0}(0)$, which is tied to the mass accretion rate onto the central object, as shown in Sect. 4 below.

\section{First-order flow}

\subsection{Upstream and downstream regions}

The $r$ - and $\theta$-components of Euler's equation, in nondimensional form, are

$u_{r} \frac{\partial u_{r}}{\partial r}+\frac{u_{\theta}}{r} \frac{\partial u_{r}}{\partial \theta}-\frac{u_{\theta}^{2}}{r}=-\frac{1}{\rho} \frac{\partial \rho}{\partial r}-\frac{1}{r^{2}}$

$u_{r} \frac{\partial u_{\theta}}{\partial r}+\frac{u_{\theta}}{r} \frac{\partial u_{\theta}}{\partial \theta}+\frac{u_{r} u_{\theta}}{r}=-\frac{1}{\rho r} \frac{\partial \rho}{\partial \theta}$.

Our procedure is first to express $u_{r}$ and $u_{\theta}$ in terms of $\psi$ and $\rho$, using Eqs. (2) and (3). We then expand $\psi$ and $\rho$ themselves in their respective perturbation series and equate the coeffcients of various powers of $r$. To avoid cumbersome division by the series for $\rho$, we first multiply Eqs. (8) and (9) through by $\rho^{3}$.

Equating the coefficients of the highest power of $r$, which is $r^{-1}$, we find that they are identically equal. Equating coefficients of $r^{-2}$ yields the first-order equations. From Eq. (8), we find

$$
\begin{aligned}
-\beta f_{1}^{\prime \prime}-\beta f_{1} & +\beta^{2} \sin \theta \cos \theta g_{-1}^{\prime} \\
& +\left(\beta^{2} \cos ^{2} \theta-1\right) g_{-1}+1=0,
\end{aligned}
$$

while Eq. (9) yields

$$
\left(1-\beta^{2} \sin ^{2} \theta\right) g_{-1}^{\prime}-\beta^{2} \sin \theta \cos \theta g_{-1}=0 .
$$

These equations are identical in form to Eqs. (18) and (19), respectively, of Paper I, hereafter designated Eqs. (I.18) and (I.19). However, their solutions may differ. The factor $\left(1-\beta^{2} \sin ^{2} \theta\right)$ in Eq. (11), which was positive in the subsonic case for any angle $\theta$, can now be positive, negative, or zero.

Consider first the upstream region. For $\theta>\theta_{M}^{\prime}$, the term $\left(1-\beta^{2} \sin ^{2} \theta\right)$ is indeed positive, and we may recast Eq. (11) as

$$
\frac{\mathrm{d}}{\mathrm{d} \theta}\left[\left(1-\beta^{2} \sin ^{2} \theta\right)^{1 / 2} g_{-1}\right]=0
$$

A84, page 4 of 17 whose solution is

$$
g_{-1}=\frac{C}{\left(1-\beta^{2} \sin ^{2} \theta\right)^{1 / 2}} .
$$

Here, $C$ is independent of $\theta$, but is possibly a function of $\beta$. The analogous result appeared in our subsonic analysis as Eq. (I.20). In that case, we ultimately found $C$ to be unity.

Returning to the supersonic flow, we see that, for any nonzero $C$, the function $g_{-1}$ diverges at $\theta=\theta_{M}^{\prime}$. This fact does not mean that the density itself diverges at that location; there is no reason for it to do so. Rather, it is our series expansion of $\rho(r, \theta)$ that fails in this region. The issue here is mathematical, rather than physical. Although the function $g_{-1}(\theta)$ diverges, the associated term in the perturbation expansion, Eq. (7), is multiplied by $r^{-1}$. At any finite angular separation from $\theta_{M}^{\prime}$, the first-order correction to the background density can be made arbitrarily small by considering a sufficiently large $r$-value. This observation suggests that the divergences encountered here and elsewhere in our analysis will vanish if we change independent variables from $r$ and $\theta$ to another set that mixes the two. In any case, we do not explore that possibility in the present paper.

Substitution of Eq. (12) for $g_{-1}$ into (10) yields the governing equation for $f_{1}$ :

$f_{1}^{\prime \prime}+f_{1}=\frac{1}{\beta}-\frac{C\left(1-\beta^{2}\right)}{\beta\left(1-\beta^{2} \sin ^{2} \theta\right)^{3 / 2}}$.

This equation, being identical to (I.21), has the same general solution:

$f_{1}=\frac{1}{\beta}-\frac{C\left(1-\beta^{2} \sin ^{2} \theta\right)^{1 / 2}}{\beta}+D \cos \theta+E \sin \theta$,

where $D$ and $E$ are additional constants. The vanishing of $f_{1}(\pi)$ tells us that $D=(1-C) / \beta$, while $f_{1}^{\prime}(\pi)=0$ implies that $E=0$. Both relations also held in the subsonic case.

We next turn to the downstream region. Here again, the critical term $\left(1-\beta^{2} \sin ^{2} \theta\right)$ is positive. The solutions for $g_{-1}$ and $f_{1}$ are thus identical to the upstream solutions in Eqs. (12) and (14), if we replace $C, D$, and $E$ by new constants $C^{\prime}, D^{\prime}$, and $E^{\prime}$. Application of the appropriate boundary conditions then tells us that $E^{\prime}=0$ and $D^{\prime}=\left(C^{\prime}-1\right) / \beta$.

Referring again to Fig. 3, the supersonic flow we are now analyzing differs from the subsonic one by the presence of the intermediate region, lying between the Mach and anti-Mach cones. As $\beta$ decreases to unity from above, this region narrows symmetrically about $\theta=\pi / 2$ and ultimately vanishes, as does the physical distinction between the supersonic and subsonic flows. Continuity strongly suggests that, while $(C, D)$ and $\left(C^{\prime}, D^{\prime}\right)$ could, in principle, be $\beta$-dependent, they are actually not. Instead, they have the truly constant values $(1,0)$ found in the subsonic case.

In summary, the first-order flow in both the upstream and downstream regions is described by

$f_{1}=\frac{1-\left(1-\beta^{2} \sin ^{2} \theta\right)^{1 / 2}}{\beta}$
$g_{-1}=\frac{1}{\left(1-\beta^{2} \sin ^{2} \theta\right)^{1 / 2}} \cdot$

After substituting these coefficients into the series expansions for $\psi$ and $\rho$, one may calculate $u_{r}$ and $u_{\theta}$ to linear order from 
Eqs. (2) and (3), respectively. At any fixed, $r$-value, however, the series representation for $\rho$ fails sufficiently close to either Mach cone, and the velocity components cannot be obtained in this manner.

Comparing our results thus far with the previous literature, Dokuchaev (1964); Ruderman \& Spiegel (1971); and Ostriker (1999) all obtained Eq. (12) for the downstream, first-order density distribution, but with $C=2$. They also found that $g_{-1}$ vanishes for $\theta>\theta_{M}$. In the subsonic analysis of Ostriker (1999), $g_{-1}$ is everywhere finite. Thus, the density distributions in her supersonic and subsonic flows do not approach one another in the $\beta=1$ limit. Ruderman \& Spiegel (1971) further argued that the apparent divergence in the downstream density perturbation at $\theta=\theta_{M}$ signifies the presence of a bowshock. While a bowshock certainly arises relatively close to the gravitating mass, it does not persist into the far field, the only regime where an analysis based on small perturbations of the background gas is justified.

\subsection{Intermediate region}

For $\theta_{M}<\theta<\theta_{M}^{\prime}$, the term $\left(1-\beta^{2} \sin ^{2} \theta\right)$ in Eq. (11) is negative. We therefore rewrite this equation as

$\left(\beta^{2} \sin ^{2} \theta-1\right) g_{-1}^{\prime}+\beta^{2} \sin \theta \cos \theta g_{-1}=0$.

This is equivalent to

$\frac{\mathrm{d}}{\mathrm{d} \theta}\left[\left(\beta^{2} \sin ^{2} \theta-1\right)^{1 / 2} g_{-1}\right]=0$

which in turn implies that

$g_{-1}=\frac{C}{\left(\beta^{2} \sin ^{2} \theta-1\right)^{1 / 2}}$.

Here we are reverting to our original notation for the integration constants, as the previous ones have all been evaluated.

Substitution of the new expression for $g_{-1}$ into Eq. (10) yields

$f_{1}^{\prime \prime}+f_{1}=\frac{1}{\beta}-\frac{C\left(\beta^{2}-1\right)}{\beta\left(\beta^{2} \sin ^{2} \theta-1\right)^{3 / 2}}$.

We may solve this equation using the method of variation of parameters. Adding the two homogeneous solutions yields the general result

$f_{1}=\frac{1}{\beta}+\frac{C\left(\beta^{2} \sin ^{2} \theta-1\right)^{1 / 2}}{\beta}+D \cos \theta+E \sin \theta$.

As in the upstream and downstream regions, the series representation of $\psi$ is well-behaved at either Mach cone, at least to linear order. Approaching either cone from the outside, Eq. (15) tells us that $f_{1} \rightarrow 1 / \beta$. Equation (20) above implies that $f_{1}$ has the additional term $D \cos \theta+E \sin \theta$ when we approach the cones from the intermediate region.

To proceed, we consider the physical interpretation of the stream function. Equation (2) tells us that $\psi(r, \theta)$ is the $\theta$-integral of the mass flux $\rho u_{r}$ over a surface of radius $r$. More generally, the stream function is the net rate of mass transport into any surface of revolution extending from $\theta=0$ to the angle of interest. A discontinuity in $\psi$ at an angle $\theta$ thus represents a thin sheet of mass being injected or ejected along the corresponding cone.
If we are to reject such a solution as unphysical, then we must demand continuity of $\psi$. That is, $f_{1}$ must again approach $1 / \beta$ at the two Mach cones, and $D=E=0$ in Eq. (20).

Our mathematical description of the first-order flow in the intermediate region is now

$f_{1}=\frac{1+C\left(\beta^{2} \sin ^{2} \theta-1\right)^{1 / 2}}{\beta}$
$g_{-1}=\frac{C}{\left(\beta^{2} \sin ^{2} \theta-1\right)^{1 / 2}}$.

Since the intermediate region does not reach either the upstream or downstream axis, we cannot appeal to our usual boundary conditions in order to evaluate $C$. We defer this issue until our analysis of the second-order flow in Sect. 4, when we will show that the requirement of mass continuity again settles the matter. For any $C$-value, the divergence of $g_{-1}$ at the Mach and antiMach cones indicates a breakdown of the series representation for the density.

\subsection{Vorticity}

A key, simplifying property of the flow is that it is irrotational. To reprise the argument from Paper I, we first write Euler's equation in the steady state as

$\boldsymbol{u} \times \boldsymbol{\omega}=\boldsymbol{\nabla} B$

where $\omega \equiv \boldsymbol{\nabla} \times \boldsymbol{u}$ is the vorticity and the Bernoulli function on the right-hand side is

$B \equiv \frac{1}{2} u^{2}+\ln \rho-\frac{1}{r}$.

Dotting both sides of Eq. (23) with $\boldsymbol{u}$, we find that

$(\boldsymbol{u} \cdot \boldsymbol{\nabla}) B=0$,

which is the familiar statement that $B$ is constant along streamlines. Moreover, $B$ approaches $\beta^{2} / 2$ at large $r$, both upstream and downstream. (Recall that the non-dimensional density $\rho$ becomes unity in this limit.) In these regions, therefore, $B$ has the same value on every streamline, and $\boldsymbol{\nabla} B=0$. Since $\omega$ is orthogonal to $\boldsymbol{u}$ in our poloidal flow, Eq. (23) imples that $\omega=0$, i.e., the flow is irrotational outside the Mach cones.

If our derived intermediate solution is correct, then irrotationality must hold there as well, since there is no physical barrier between the intermediate flow and those upstream and downstream, at least in the laminar, far field. The only non-zero component of the vorticity is $\omega_{\phi}$, so the condition of irrotationality becomes

$\frac{\partial u_{r}}{\partial \theta}=\frac{\partial\left(r u_{\theta}\right)}{\partial r}$

To test the validity of this relation in the intermediate region, we use Eqs. (2) and (3) for $u_{r}$ and $u_{\theta}$, respectively, and then substitute in the series expansions for $\psi$ and $\rho$, Eqs. (6) and (7).

Equating the coefficient of the highest power of $r$, which is $r^{0}$, is equivalent to testing for irrotationality in the uniform, background flow. Specifically, we require

$f_{2}^{\prime \prime}-f_{2}^{\prime} \cot \theta=-2 f_{2}$. 
Using $f_{2}=\beta \sin ^{2} \theta / 2$, we verify that the above equation does hold. This result is to be expected, as a uniform flow is manifestly irrotational.

We next equate the coefficients of $r^{-1}$, effectively testing irrotationality in the first-order flow. The required condition is now

$f_{1}^{\prime \prime}-f_{2}^{\prime \prime} g_{-1}-f_{2}^{\prime} g_{-1}^{\prime}=\left(f_{1}^{\prime}-f_{2}^{\prime} g_{-1}\right) \cot \theta$,

where $f_{1}$ and $g_{-1}$ are given by Eqs. (21) and (22), respectively. Using these functional forms, we find that this last equation is satisfied for any value of $C$. Thus, the first-order flow in the intermediate region is indeed irrotational.

\section{Second-order flow}

\subsection{Dynamical equations}

Having established the first-order flow, at least up to the constant $C$, we consider the next higher approximation. We return to the perturbative expansion of Euler's equation, as described at the beginning of Sect. 3.1. By equating coefficients of $r^{-3}$, we derive the second-order equations, which govern the variables $f_{0}$ and $g_{-2}$. Here the source terms involve $f_{1}$ and $g_{-1}$. Prior to substituting in the explicit solutions for $f_{1}$ and $g_{-1}$, the equations are identical to those in the subsonic problem; we display them again for convenient reference.

From the $r$-component of Euler's equation, we derive Eq. (I.27), which is

$$
\begin{aligned}
-\beta f_{0}^{\prime \prime}-\beta \cot \theta & f_{0}^{\prime}+\beta^{2} \sin \theta \cos \theta g_{-2}^{\prime} \\
& +\left(2 \beta^{2} \cos ^{2} \theta-2\right) g_{-2}=\mathcal{A}_{1}+\mathcal{A}_{2}+\mathcal{A}_{3} .
\end{aligned}
$$

The three right-hand terms are

$$
\begin{aligned}
\mathcal{A}_{1} \equiv & \frac{f_{1}^{2}}{\sin ^{2} \theta}-\frac{f_{1} f_{1}^{\prime} \cos \theta}{\sin ^{3} \theta}+\frac{\left(f_{1}^{\prime}\right)^{2}}{\sin ^{2} \theta}+\frac{f_{1} f_{1}^{\prime \prime}}{\sin ^{2} \theta} \\
\mathcal{A}_{2} \equiv & \beta f_{1} g_{-1}-2 \beta f_{1}^{\prime} g_{-1} \cot \theta-\beta f_{1} g_{-1}^{\prime} \cot \theta-\beta f_{1}^{\prime} g_{-1}^{\prime} \\
& +\beta f_{1}^{\prime \prime} g_{-1} \\
\mathcal{A}_{3} \equiv & 2 g_{-1}^{2}-3 g_{-1} .
\end{aligned}
$$

The $\theta$-component of Euler's equation yields Eq. (I.31):

$$
-\beta f_{0}^{\prime}+\mathcal{D} g_{-2}^{\prime}-2 \beta^{2} \sin \theta \cos \theta g_{-2}=\mathcal{B}_{1}+\mathcal{B}_{2}+\mathcal{B}_{3} \text {. }
$$

Here,

$\mathcal{D} \equiv 1-\beta^{2} \sin ^{2} \theta$,

and

$$
\begin{aligned}
& \mathcal{B}_{1} \equiv f_{1}^{2} \cot \theta-\frac{f_{1} f_{1}^{\prime}}{\sin ^{2} \theta} \\
& \mathcal{B}_{2} \equiv \beta f_{1} g_{-1} \cot \theta+\beta f_{1}^{\prime} g_{-1}+2 \beta f_{1} g_{-1}^{\prime} \\
& \mathcal{B}_{3} \equiv-2 g_{-1} g_{-1}^{\prime} .
\end{aligned}
$$

In the upstream and downstream regions, there are no unknown constants in the source terms, i.e., both $f_{1}$ and $g_{-1}$ are identical to their subsonic counterparts. Hence, the explicit form of the second-order equations is also the same. Referring to Eqs. (I.35) and (I.36), we have

$$
\begin{aligned}
-\beta f_{0}^{\prime \prime} & -\beta \cot \theta f_{0}^{\prime}+\beta^{2} \sin \theta \cos \theta g_{-2}^{\prime} \\
& +\left(2 \beta^{2} \cos ^{2} \theta-2\right) g_{-2}=\frac{1}{\mathcal{D}}-\frac{3}{\sqrt{\mathcal{D}}}+\frac{2}{1+\sqrt{\mathcal{D}}},
\end{aligned}
$$

and

$$
\begin{aligned}
-\beta f_{0}^{\prime} & +\mathcal{D} g_{-2}^{\prime}-2 \beta^{2} \sin \theta \cos \theta g_{-2} \\
& =\beta^{2} \sin \theta \cos \theta\left[-\frac{2}{\mathcal{D}^{2}}+\frac{2}{\mathcal{D}^{3 / 2}}-\frac{1}{\mathcal{D}}+\frac{1}{(1+\sqrt{\mathcal{D}})^{2}}\right]
\end{aligned}
$$

In the intermediate region, however, $f_{1}$ and $g_{-1}$ are given by the new expressions in Eqs. (21) and (22), respectively. After lengthy manipulation, we find the new source terms, and hence the explicit dynamical equations in this region. These equations, which still contain the unknown constant $C$, are

$-\beta f_{0}^{\prime \prime}-\beta \cot \theta f_{0}^{\prime}+\beta^{2} \sin \theta \cos \theta g_{-2}^{\prime}+\left(2 \beta^{2} \cos ^{2} \theta-2\right) g_{-2}=$

$\frac{2 C \mathcal{E}^{1 / 2}}{\beta^{2} \sin ^{2} \theta}-\frac{3 C}{\mathcal{E}^{1 / 2}}+\frac{C^{2}}{\mathcal{E}}+\frac{1-C^{2}}{\beta^{2} \sin ^{2} \theta}$,

and

$-\beta f_{0}^{\prime}-\mathcal{E} g_{-2}^{\prime}-2 \beta^{2} \sin \theta \cos \theta g_{-2}=$

$\frac{2 C^{2} \beta^{2} \sin \theta \cos \theta}{\mathcal{E}^{2}}-\frac{2 C \beta^{2} \sin \theta \cos \theta}{\mathcal{E}^{3 / 2}}$

$-\frac{C^{2} \beta^{2} \sin \theta \cos \theta}{\mathcal{E}}+\frac{\left(1-C^{2}\right) \cot \theta}{\beta^{2} \sin ^{2} \theta}$

$+C^{2} \cot \theta+\frac{2 C \mathcal{E}^{1 / 2} \cot \theta}{\beta^{2} \sin ^{2} \theta}$

Here, we have defined

$\mathcal{E} \equiv \beta^{2} \sin ^{2} \theta-1$,

which is positive throughout this region.

\subsection{Near-cone divergence}

Our plan is to integrate numerically two, coupled second-order equations in order to determine the variables $f_{0}^{\prime}$ and $g_{-2}$ throughout the flow. With $f_{0}^{\prime}$ in hand, another integration will yield $f_{0}$ itself. Before we embark on this program, let us consider more carefully the behavior of $f_{0}^{\prime}$ and $g_{-2}$. In the upstream and downstream regions, the term $\mathcal{D}$ vanishes as one approaches either Mach cone. Thus, the source terms in Eqs. (31) and (32) diverge in that limit. Similarly, the source terms in Eqs. (33) and (34) diverge because of the vanishing of $\mathcal{E}$. It is possible, then, that both $f_{0}^{\prime}$ and $g_{-2}$ also diverge at the cones.

This is indeed the case. In this section, we first establish the divergences of $f_{0}^{\prime}$ and $g_{-2}$ in the downstream region only. That is, we assume that $\theta$ approaches $\theta_{M}$ from below. Later in the section, we outline the derivation for the remaining regions of the flow. We then use the full result to shed light on the unknown parameter $C$.

Since $\theta<\theta_{M}$, we define the positive angle $\alpha \equiv \theta_{M}-\theta$, and assume that $f_{0}^{\prime}$ and $g_{-2}$ take the following asymptotic forms as $\alpha$ diminishes:

$f_{0}^{\prime}=\zeta \alpha^{-m}$

$g_{-2}=\gamma \alpha^{-n}$, 
where $\zeta, \gamma, m$, and $n$ are all functions of $\beta$ only. If both $f_{0}^{\prime}$ and $g_{-2}$ truly diverge, then $m$ and $n$ are positive. Within Eqs. (31) and (32), we replace $\sin \theta$ and $\cos \theta$ by their limiting values, $1 / \beta$ and $\sqrt{\beta^{2}-1} / \beta$, respectively. We further note that $\mathcal{D} \rightarrow$ $2 \alpha \sqrt{\beta^{2}-1}$. After differentiating the asymptotic forms of $f_{0}^{\prime}$ and $g_{-2}$ with respect to $\theta$, we derive simplified, limiting forms of Eqs. (31) and (32). Expressed using $\alpha$ as the independent variable, these are

$$
\begin{aligned}
& -m \beta \zeta \alpha^{-m-1}+n \gamma \sqrt{\beta^{2}-1} \alpha^{-n-1}=-\frac{\beta^{2}}{4\left(\beta^{2}-1\right)} \alpha^{-1} \\
& \beta \zeta \alpha^{-m}+2(1-n) \gamma \sqrt{\beta^{2}-1} \alpha^{-n}=\frac{1}{2 \sqrt{\beta^{2}-1}} \alpha^{-2} .
\end{aligned}
$$

In deriving these equations, we have retained only the most rapidly diverging terms on the right-hand sides of Eqs. (31) and (32). We have also dropped terms proportional to $\alpha^{-m}$ and $\alpha^{-n}$ in the left-hand side of Eq. (31), since both terms are dominated, in the small- $\alpha$ limit, by the two we have kept.

We next consider the $\beta$-dependence of $m$ and $n$. The righthand side of Eq. (39) is proportional to $\alpha^{-2}$. For this equation to balance as $\alpha$ diminishes, one left-hand term could also diverge as $\alpha^{-2}$, and the other more slowly. However, if this were the case, i.e., if either $m=2>n$ or $n=2>m$, then Eq. (38) would be unbalanced. It is also posssible that the two left-hand terms in (39) diverge more rapidly than $\alpha^{-2}$, balancing each other. We would then have $m=n>2$. Finally, all three terms in Eq. (39) could diverge at the same rate: $m=n=2$.

Assuming provisionally that $m=n>2$, then we may ignore the right-hand terms of both Eqs. (38) and (39) in the asymptotic limit. After dividing out all terms containing $\alpha$, the two equations reduce to

$-\beta \zeta+\gamma \sqrt{\beta^{2}-1}=0$

$\beta \zeta+2(1-n) \gamma \sqrt{\beta^{2}-1}=0$.

Adding these two yields

$(3-2 n) \gamma \sqrt{\beta^{2}-1}=0$,

which implies that $n=3 / 2$. Since we assumed $n>2$ at the outset, our original hypothesis, $m=n>2$, was incorrect.

We have established that $m=n=2$. Equations (38) and (39) now reduce asymptotically to

$$
\begin{aligned}
& -\beta \zeta+\gamma \sqrt{\beta^{2}-1}=0 \\
& \beta \zeta-2 \gamma \sqrt{\beta^{2}-1}=\frac{1}{2 \sqrt{\beta^{2}-1}},
\end{aligned}
$$

which have the unique solution

$$
\begin{aligned}
& \zeta=\frac{-1}{2 \beta \sqrt{\beta^{2}-1}} \\
& \gamma=\frac{-1}{2\left(\beta^{2}-1\right)} .
\end{aligned}
$$

Consider next the region just upstream from the Mach cone. Here, we may assume the same asymptotic forms for $f_{0}^{\prime}$ and $g_{-2}$ as in Eqs. (36) and (37), provided the independent variable $\alpha$ remains positive: $\alpha \equiv \theta-\theta_{M}$. Inserting these functional forms into the second-order Eqs. (33) and (34), we note that $\mathrm{d} \alpha / \mathrm{d} \theta$ changes sign from -1 to +1 , and that $\mathcal{E}$ now approaches $2 \alpha \sqrt{\beta^{2}-1}$ near the cone. We then derive equations analogous to (38) and (39), which may again be solved in the near-cone limit. After applying similar reasoning to the two regions surrounding the anti-Mach cone, we arrive at the following asymptotic forms for $f_{0}^{\prime}$ and $g_{-2}$ near $\theta_{M}$ and $\theta_{M}^{\prime}$ :

$$
\begin{aligned}
f_{0}^{\prime} & =\frac{-1}{2 \beta \sqrt{\beta^{2}-1}\left(\theta_{M}-\theta\right)^{2}} & & \theta \lesssim \theta_{M} \\
& =\frac{C^{2}}{2 \beta \sqrt{\beta^{2}-1}\left(\theta-\theta_{M}\right)^{2}} & & \theta \gtrsim \theta_{M} \\
& =\frac{-C^{2}}{2 \beta \sqrt{\beta^{2}-1}\left(\theta_{M}^{\prime}-\theta\right)^{2}} & & \theta \lesssim \theta_{M}^{\prime} \\
& =\frac{1}{2 \beta \sqrt{\beta^{2}-1}\left(\theta-\theta_{M}^{\prime}\right)^{2}} & & \theta \gtrsim \theta_{M}^{\prime},
\end{aligned}
$$

and

$$
\begin{aligned}
g_{-2} & =\frac{-1}{2\left(\beta^{2}-1\right)\left(\theta_{M}-\theta\right)^{2}} & & \theta \lesssim \theta_{M} \\
& =\frac{C^{2}}{2\left(\beta^{2}-1\right)\left(\theta-\theta_{M}\right)^{2}} & & \theta \gtrsim \theta_{M} \\
& =\frac{C^{2}}{2\left(\beta^{2}-1\right)\left(\theta_{M}^{\prime}-\theta\right)^{2}} & & \theta \lesssim \theta_{M}^{\prime} \\
& =\frac{-1}{2\left(\beta^{2}-1\right)\left(\theta-\theta_{M}^{\prime}\right)^{2}} & & \theta \gtrsim \theta_{M}^{\prime} .
\end{aligned}
$$

Obtaining $f_{0}$ requires that we integrate $f_{0}^{\prime}$ over $\theta$. Starting at the upstream axis, with $f_{0}(\pi)=0$, the integrated $f_{0}(\theta)$ diverges to positive infinity as $\theta$ approaches $\theta_{M}^{\prime}$. However, the stream function has a direct physical meaning as the mass transfer rate into a surface of revolution. Since this rate is finite for any $\theta$, the function $f_{0}^{\prime}(\theta)$ must be integrable across the anti-Mach cone. That is, the upward divergence of $f_{0}^{\prime}$ for $\theta \gtrsim \theta_{M}^{\prime}$ must be cancelled by a matching downward divergence for $\theta \lesssim \theta_{M}^{\prime}$. A similar antisymmetry of the divergences must occur at the Mach cone, $\theta=\theta_{M}$ (see Fig. 4). Inspection of Eq. (40) shows that this requirement forces $C^{2}$ to be unity. The parameter $C$ itself is either +1 or -1 , independent of $\beta$. We will demonstrate presently that the positive solution is the physically relevant one.

\subsection{Vorticity}

The second-order flow must be irrotational, as we argued in Sect. 3.3. Starting with Eq. (25), we again replace the velocity components by $\psi$ and $\rho$, and develop the latter in our perturbation series. Equating coefficients of $r^{-2}$ tests irrotationality in the second-order flow. Specifically, we require

$$
\begin{aligned}
& \left(-f_{2}^{\prime} g_{-1}^{2}+f_{2}^{\prime} g_{-2}+f_{1}^{\prime} g_{-1}-f_{0}^{\prime}\right) \cot \theta \\
& +f_{2}^{\prime \prime} g_{-1}^{2}+2 f_{2}^{\prime} g_{-1} g_{-1}^{\prime}-f_{2}^{\prime \prime} g_{-2} \\
& -f_{2}^{\prime} g_{-2}^{\prime}-f_{1}^{\prime \prime} g_{-1}-f_{1}^{\prime} g_{-1}^{\prime}+f_{0}^{\prime \prime} \\
& =2 f_{2} g_{-2}^{2}-2 f_{2} g_{-2}-f_{1} g_{-1} .
\end{aligned}
$$

Within the upstream and downstream regions, Eqs. (15) and (16) give us $f_{1}$ and $g_{-1}$, respectively. Substituting these functions into Eq. (42) then yields the condition of irrotationality:

$$
\begin{aligned}
\beta f_{0}^{\prime \prime}-\beta \cot \theta f_{0}^{\prime} & -\beta^{2} \sin \theta \cos \theta g_{-2}^{\prime} \\
& +2 \beta^{2} \sin ^{2} \theta g_{-2}=\frac{1}{\mathcal{D}}-\frac{1}{\sqrt{\mathcal{D}}}
\end{aligned}
$$




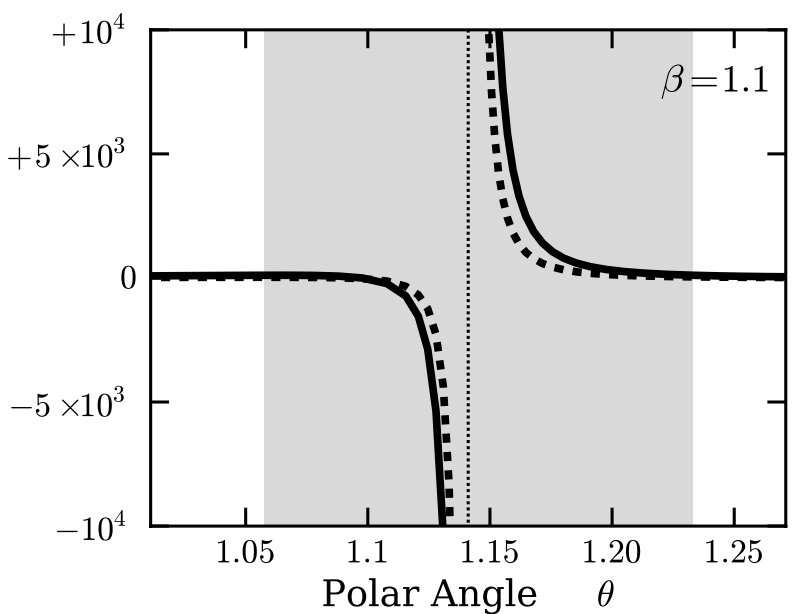

Fig. 4. Asymmetric divergences at the Mach angle, for $\beta=1.1$. The solid and dashed curves trace $f_{0}^{\prime}$ and $g_{-2}$, respectively, close to the Mach angle $\theta_{M}$, marked here by the vertical, dotted line. The functions diverge antisymmetrically once we choose $C^{2}=1$. Both curves are taken from the numerical integration outlined in Sect. 4.5. The shaded region is that which we remove before enforcing continuity of $f_{0}$ and $g_{-2}$ across the cones.

Finally, we may combine this last relation with Eq. (31), the $r$-component of Euler's equation, to obtain

$$
-2 \beta \cot \theta f_{0}^{\prime}+2\left(\beta^{2}-1\right) g_{-2}=\frac{2}{\mathcal{D}}-\frac{4}{\sqrt{\mathcal{D}}}+\frac{2}{1+\sqrt{\mathcal{D}}} .
$$

This is identical to Eq. (I.64), and will prove useful when we evaluate the force.

Turning to the intermediate region, we need to use Eqs. (21) and (22) for $f_{1}, g_{-1}$, and their derivatives. In both equations, the unknown parameter $C$ appears. Substitution into Eq. (42) above yields the condition for irrotationality:

$$
\begin{aligned}
\beta f_{0}^{\prime \prime} & -\beta \cot \theta f_{0}^{\prime}-\beta^{2} \sin \theta \cos \theta g_{-2}^{\prime} \\
& +2 \beta^{2} \sin ^{2} \theta g_{-2}=\frac{C^{2}}{\mathcal{E}}-\frac{C}{\sqrt{\mathcal{E}}}=\frac{1}{\mathcal{E}}-\frac{C}{\sqrt{\mathcal{E}}} .
\end{aligned}
$$

In the last form of this equation, we have used the fact that $C^{2}=1$. Combination with the Euler Eq. (31) gives

$$
\begin{aligned}
-2 \beta \cot \theta f_{0}^{\prime} & +2\left(\beta^{2}-1\right) g_{-2} \\
& =\frac{2 C^{2}}{\mathcal{E}}-\frac{4 C}{\mathcal{E}^{1 / 2}}+\frac{1-C^{2}}{\beta^{2} \sin ^{2} \theta}+\frac{2 C \mathcal{E}^{1 / 2}}{\beta^{2} \sin ^{2} \theta} \\
& =\frac{2}{\mathcal{E}}-\frac{4 C}{\mathcal{E}^{1 / 2}}+\frac{2 C \mathcal{E}^{1 / 2}}{\beta^{2} \sin ^{2} \theta}
\end{aligned}
$$

which will again aid in the force evaluation.

We emphasize a key difference between Eqs. (44) and (46). Because the upstream and downstream regions join smoothly onto the uniform background, the flow in both is guaranteed to be irrotational to all orders. In our numerical determination of the flow, to be described in Sect. 4.5 below, we verified that Eq. (44) indeed holds numerically both upstream and downstream. In contrast, we need to impose Eq. (46), or an equivalent condition, to obtain a physically acceptable solution in the intermediate region.

Enforcing irrotationality in the intermediate region requires only that we impose this condition along any interior cone, i.e., at a $\theta$-value such that $\theta_{M}<\theta<\theta_{M}^{\prime}$. To see why, return to Euler's

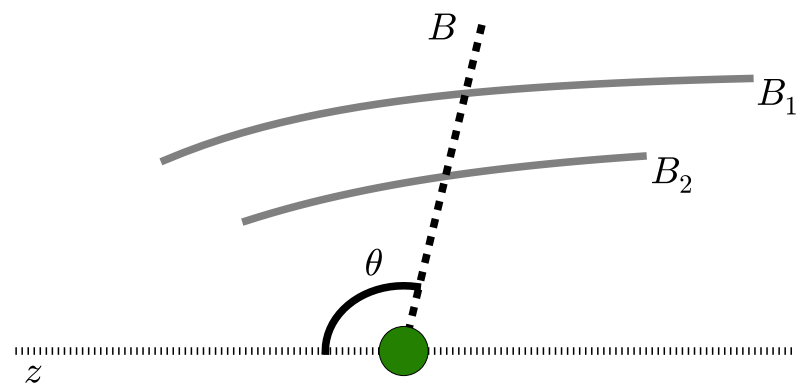

Fig. 5. Enforcing irrotationality. In our steady-state, isothermal flow, the Bernoulli function $B$ is constant along each streamline. In principle, $B$ could vary from one streamline to the next. However, if we also make $B$ constant along any cone, then the function is a universal constant and the flow is irrotational.

equation, in the form given by Eq. (23). Projecting this vector equation along the $r$-direction gives

$u_{\theta} \omega_{\phi}=\frac{\partial B}{\partial r}$.

This relation holds at fixed $\theta$ and $\phi$. Since the flow is axisymmetric, the equation also holds at all $\phi$-values, i.e., along a cone. If $\omega_{\phi}$ vanishes along such a cone, then $B$ is constant on that surface: $\partial B / \partial r=0$. But we already know that $B$ does not vary along any streamline. As Fig. 5 illustrates schematically, the additional constraint tells us that the $B$-values characterizing each streamline are identical, i.e., $B$ is a true spatial constant. Thus, $\nabla B=0$ and, from Eq. (23), the flow is irrotational.

In practice, we apply $\omega_{\phi}=0$ at $\theta=\pi / 2$, the equatorial plane of the flow. The irrotationality condition, expressed as Eq. (46), implies that there is a unique (but $\beta$-dependent) value of $g_{-2}$ at that angle:

$g_{-2}(\pi / 2)=\frac{2}{\left(\beta^{2}-1\right)^{2}}-\frac{2 C}{\left(\beta^{2}-1\right)^{3 / 2}}+\frac{C}{\beta\left(\beta^{2}-1\right)^{1 / 2}}$.

When we find the flow numerically in the intermediate region, we will impose Eq. (47) as an initial condition. The resulting flow is then irrotational. That is, all solutions of Euler's Eqs. (33) and (34) also obey Eq. (46).

\subsection{Mass accretion}

It is only in the second-order flow that mass accretion onto the central object appears. Moreover, the function $f_{0}(\theta)$, evaluated on the downstream axis, gives the actual accretion rate. Higherorder approximations to the flow contribute no additional information in this regard. We now review the argument, first advanced in Paper I, and apply it to the present, supersonic, case.

Consider the net transfer rate of mass into a sphere of radius $r$. In a steady-state flow, this rate is the same through any closed surface; we simply use a sphere for convenience. The dimensional result is

$\dot{M}=-2 \pi \int_{0}^{\pi} \rho u_{r} r^{2} \sin \theta \mathrm{d} \theta=2 \pi \psi(r, 0)$.

Here we have substituted Eq. (2) for $u_{r}$ and utilized the normalization for the stream function, $\psi(r, \pi)=0^{3}$. We have also implicitly assumed that $\rho$ and $u_{r}$ are smooth functions, despite the divergences arising in their perturbation expansions.

3 By symmetry, the upstream axis coincides with a streamline, so that $\psi(r, \pi)$ is a constant. Such an additive constant does not effect any physical result, so we have set it to zero. 
If we identify $2 \pi \rho_{0} c_{\mathrm{s}} r_{\mathrm{s}}^{2}$ as our fiducial mass accretion rate, then the non-dimensional counterpart of the last equation becomes

$\dot{M}=\psi(r, 0)$.

Substitution of the series expansion for $\psi(r, 0)$ in Eq. (6) and application of the boundary condition that $f_{i}(0)=0$ for $i=$ $1,-1,-2$, etc. leads to

$\dot{M}=f_{0}(0)$.

It is noteworthy that $\dot{M}$ depends only on a coefficient from the second-order expansion; this fact calls for a more physical explanation. One reason is that the streamlines of the first-order flow are symmetric, so that accretion does not occur to this approximation. Secondly, the mass flux $\rho u_{r}$, when expanded using approximations higher than second-order, generates terms that fall off faster than $r^{-2}$. After integrating these terms over a large bounding sphere and taking the large- $r$ limit, they do not contribute to $\dot{M}$. In any event, Eq. (49) underscores the fact that the function $f_{0}^{\prime}(\theta)$ must be integrable across both Mach cones, despite its divergent behavior at the cones themselves.

We may now use our second-order equations to obtain a useful relation between $f_{0}(0)$, and hence the mass accretion rate, and the flow density. Consider first the upstream and downstream regions. The left-hand side of Eq. (32) is a perfect derivative:

$$
\begin{aligned}
-\beta f_{0}^{\prime}+\mathcal{D} g_{-2}^{\prime} & -2 \beta^{2} \sin \theta \cos \theta g_{-2} \\
& =\frac{\mathrm{d}}{\mathrm{d} \theta}\left[-\beta f_{0}+\left(1-\beta^{2} \sin \theta\right) g_{-2}\right] .
\end{aligned}
$$

In the right-hand side of the same equation, we note that $\mathcal{D}$ is an even function, in the sense that $\mathcal{D}(\theta)=\mathcal{D}(\pi-\theta)$. Since $\cos \theta$ is an odd function, $\cos \theta=-\cos (\pi-\theta)$, the entire right-hand side of Eq. (32) is odd.

Within the intermediate region, the left-hand side of Eq. (34) is

$$
\begin{aligned}
-\beta f_{0}^{\prime}-\mathcal{E} g_{-2}^{\prime} & -2 \beta^{2} \sin \theta \cos \theta g_{-2} \\
& =\frac{\mathrm{d}}{\mathrm{d} \theta}\left[-\beta f_{0}+\left(1-\beta^{2} \sin ^{2} \theta\right) g_{-2}\right] .
\end{aligned}
$$

Again, the right-hand side of Eq. (34) is odd. Now the integral of an odd function from the downstream to the upstream axes vanishes. Thus, if we integrate Eq. (32) from $\theta=0$ to $\theta_{M}$, Eq. (34) from $\theta_{M}$ to $\theta_{M}^{\prime}$, and Eq. (32) from $\theta_{M}^{\prime}$ to $\pi$, we find that

$-\beta f_{0}(0)+g_{-2}(0)=-\beta f_{0}(\pi)+g_{-2}(\pi)$.

Using $f_{0}(\pi)=0$, we have the desired result:

$f_{0}(0)=\frac{g_{-2}(0)-g_{-2}(\pi)}{\beta}$.

The same relation appeared in the subsonic study as Eq. (I.46).

\subsection{Numerical solution}

\subsubsection{Evaluation of $C$}

Our description of the flow is clearly incomplete until we identify the still unknown parameter $C$, and not just its absolute value. We again employ physical reasoning. The value of $C$ affects the shape of the streamlines, even in the first-order flow. Only one shape is reasonable dynamically. Figure 6 shows streamlines of the first-order flow for the case $\beta=1.1$. That is,

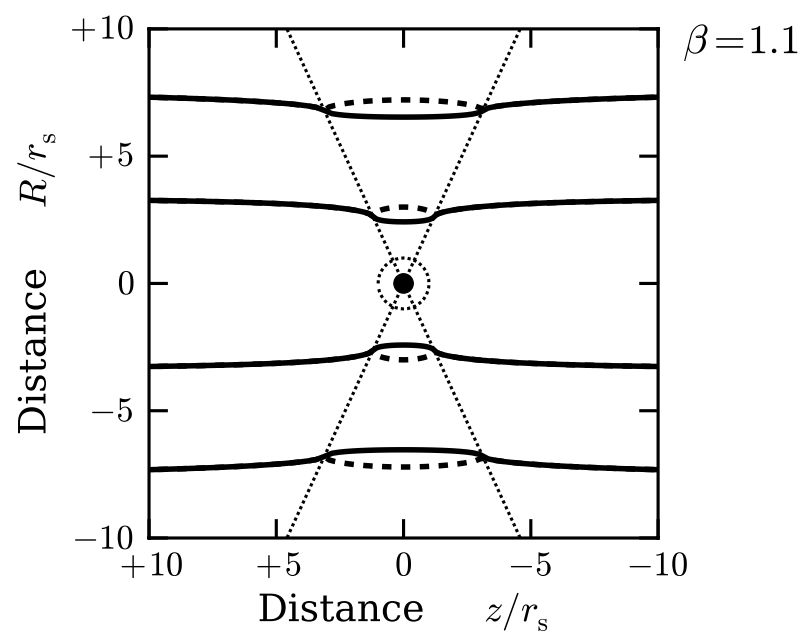

Fig. 6. First-order streamlines. Shown are contours of constant $f_{2} r^{2}+$ $f_{1} r$, for the case $\beta=1.1$. The dotted, diagonal lines trace the Mach and anti-Mach cones, while the central, dotted circle is $r / r_{\mathrm{s}}=1$. Within the intermediate region, the dashed streamlines were constructed assuming $C=-1$. The solid ones correspond to $C=+1$ and are more realistic.

we plot contours of constant $f_{2} r^{2}+f_{1} r$. In the upstream and donwstream regions, $f_{1}$ is taken from Eq. (15). For the intermediate region, we use Eq. (21), with both $C=+1$ (solid curves) and $C=-1$ (dashed curves). For either choice of the parameter, we see that all first-order streamlines are symmetric about the equatorial plane, as we found in the subsonic flow (see, e.g., Fig. I.2). The kinks at both Mach cones result from the crudeness of this first-order approximation, and would disappear in successively more accurate treatments.

The solid curves in Fig. 6 bow inward, toward the central mass, both upstream of the anti-Mach cone and inside it. Such inward turning results naturally from the gravitational pull of the mass. Eventually, the concurrent rise in density and pressure pushes the gas outward. If we were instead to choose $C=-1$, the streamlines in the intermediate region would bow outward, which is not expected.

We may also consider the matter more quantitatively by examining the velocity component $u_{R}$. Here $R$ is the cylindrical radius: $R \equiv r \sin \theta$. In the far-field limit, $u_{R}$ tends to zero, since the background flow is in the $z$-direction. Closer to the equatorial plane, but still upstream, $u_{R}$ should be slightly negative. Downstream, this velocity component should reverse sign as each streamline rejoins the background flow. Starting with the expreessions for $f_{1}$ and $g_{-1}$ in the intermediate region, we first calculate $u_{r}$ and $u_{\theta}$ from Eqs. (2) and (3), and then obtain $u_{R} \equiv u_{r} \sin \theta+u_{\theta} \cos \theta$. We find that

$u_{R}=\left[\frac{C-\mathcal{E}^{1 / 2}}{r \beta \mathcal{E}^{1 / 2}}\right] \cot \theta$.

Upstream from the mass $(\theta>\pi / 2)$, we have $\cot \theta>0$. Thus, if $C=-1$, then $u_{R}>0$ in this direction. Conversely, $u_{R}<0$ downstream from the mass. This behavior is contrary to the physically reasonable one, so we conclude that $C=+1$.

\subsubsection{Integration scheme}

Determining the second-order flow requires that we numerically integrate Eqs. (31) and (32) in the upstream and downstream regions, and Eqs. (33) and (34), with $C=+1$, in the intermediate region. In all cases, we need to specify appropriate boundary 
values of $f_{0}^{\prime}$ and $g_{-2}$. We then perform an additional integration to obtain $f_{0}$ and thereby the streamlines.

One of our boundary conditions is the value of $f_{0}(0)$. As we have seen, this quantity is also the mass accretion rate $\dot{M}$. There is still no fundamental theory to supply this rate, except at $\beta=0$ (Bondi 1952), and in the hypersonic $(\beta \gg 1)$ limit (Hoyle \& Lyttleton 1939; Bondi \& Hoyle 1944). Extending the work of Bondi (1952), Moeckel \& Throop (2009) suggested an interpolation formula that both respects the analytic limits and agrees reasonably well with numerical simulations. As we did in Paper I, we adopt this prescription, which is

$\dot{M}(\beta)=\frac{2\left(\lambda^{2}+\beta^{2}\right)^{1 / 2}}{\left(1+\beta^{2}\right)^{2}}=f_{0}(0)$.

Here, $2 \lambda=\mathrm{e}^{3 / 2} / 2=2.24$ is the analytic value of $\dot{M}(0)$ in the isothermal case (Bondi 1952).

We are now in a position to outline our numerical procedure. Starting at the upstream axis, $\theta=\pi$, we set $f_{0}(\pi)=f_{0}^{\prime}(\pi)=0$. We provisionally treat $g_{-2}(\pi)$ as a free parameter, to be determined later in order to ensure smoothness of the flow across the Mach cones, as we describe in more detail below. For any selected $g_{-2}(\pi)$, we may then integrate Eqs. (31) and (32) to the anti-Mach cone, $\theta=\theta_{M}^{\prime}$. Simultaneous integration of $f_{0}^{\prime}$ yields $f_{0}$ itself.

But knowledge of both $g_{-2}(\pi)$ and $f_{0}(0)$ also gives us $g_{-2}(0)$, according to Eq. (50). Since $f_{0}^{\prime}(0)=0$, we may again integrate the second-order Eqs. (31) and (32), along with $f_{0}^{\prime}$, from $\theta=0$ to $\theta=\theta_{M}$. At this point, we have established a one-parameter family of flows covering both the upstream and downstream regions. For any value of $g_{-2}(\pi)$, both flows are irrotational, as required physically.

Turning to the intermediate region, we begin at the equatorial plane, $\theta=\pi / 2$. We use Eq. (47) for $g_{-2}(\pi / 2)$, again setting $C=+1$. Our free parameter is now $f_{0}^{\prime}(\pi / 2)$. For any value of this quantity, we integrate Eqs. (33) and (34) away from the plane in both directions until we come to the Mach cones. We have then established the run of $f_{0}^{\prime}$ and $g_{-2}$ throughout the intermediate region. Again, the flow is irrotational for any value of $f_{0}^{\prime}(\pi / 2)^{4}$.

\subsubsection{Enforcing smoothness}

Within the intermediate region, we only know $f_{0}$ up to a constant of integration, which has yet to be fixed. In addition, we have not yet determined our two free parameters, $f_{0}^{\prime}(\pi / 2)$ and $g_{-2}(\pi)$. All three quantities are specified by requiring that the flow be smoothly varying. If $f_{0}^{\prime}$ and $g_{-2}$ were well-defined everywhere, this task would be straightforward. For example, we could tune one parameter until $g_{-2}$, as calculated in the downstream region, matched the intermediate $g_{-2}$ at the Mach cone. However, both $f_{0}^{\prime}$ and $g_{-2}$ diverge at the cones. Hence, we can only require smoothness outside some finite region surrounding each cone.

We therefore stopped each integration at a point where the divergent behavior begins to dominate. For example, when integrating from $\theta=\pi$, we examined the ratio $\alpha \equiv\left|(\pi-\theta) f_{0}^{\prime \prime} / f_{0}^{\prime}\right|$. Since $f_{0}^{\prime}(\pi)=0$, this quantity is close to unity for $\theta \lesssim \pi$. However, $\alpha$ climbs sharply near $\theta_{M}^{\prime}$. In practice, we stopped the integration at $\alpha=10$. We adopted equivalent criteria in the other

\footnotetext{
4 On a related issue, not only do the flows in all regions have spatially uniform values of the Bernoulli function $B$, but these values match: $B=\beta^{2} / 2$. To see this, we may expand Eq. (24) for $B$, using our perturbation series. The lowest-order ( $r$-independent) terms sum to $\beta^{2} / 2$ in all regions, while the higher-order terms sum to zero.
}

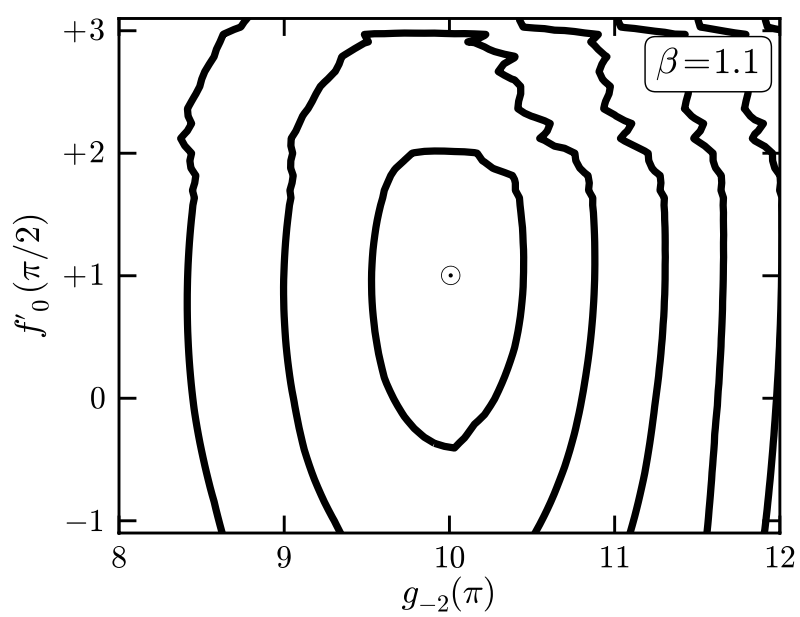

Fig. 7. Contours of constant $\chi$, where $\chi$ is defined by Eq. (52) in the text. Adjacent contours are separated by a $\chi$-interval of 15.0 . The minimum point, indicated by the central dot within the circle, is $f_{0}^{\prime}(\pi / 2)=0.96$ and $g_{-2}(\pi)=10.0$. The $\chi$-value of this point is 2.22 .

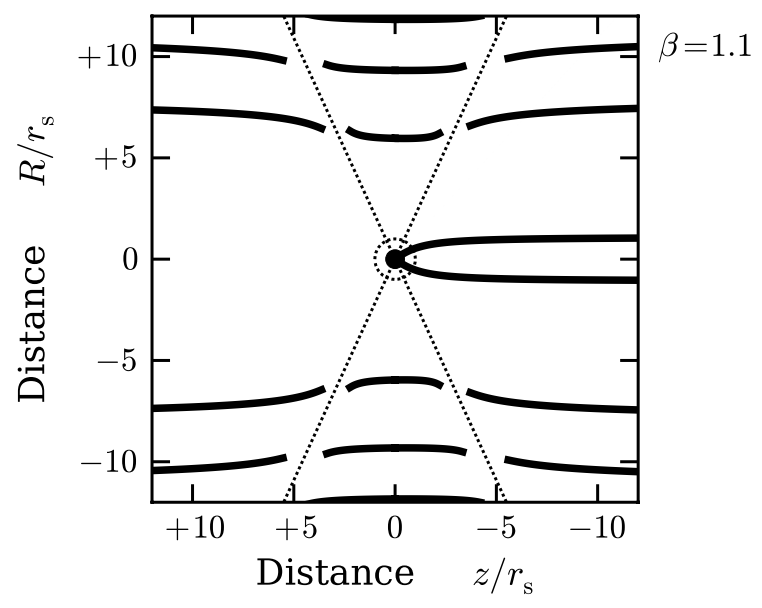

Fig. 8. Streamlines of the second-order flow, for $\beta=1.1$, constructed using the best-fit parameters from Fig. 7. The shaded regions surrounding the two Mach cones represent sectors in which the perturbation coefficients diverge. Also indicated are the central mass and the surrounding circle corresponding to $r=1$. Notice that the innermost streamlines reach the origin, indicating the occurrence of mass accretion.

regions. thus establishing boundaries for the well-behaved flow. Finally, we chose $f_{0}^{\prime}(\pi / 2)$ and $g_{-2}(\pi)$ so that $f_{0}$ and $g_{-2}$ matched as closely as possible across these boundaries.

A convenient measure of the goodness of fit is

$\chi^{2} \equiv \Delta g_{-2}^{2}\left(\theta_{M}^{\prime}\right)+\Delta f_{0}^{2}\left(\theta_{M}\right)+\Delta g_{-2}^{2}\left(\theta_{M}\right)$.

Here, $\Delta g_{-2}\left(\theta_{M}^{\prime}\right)$ is difference of $g_{-2}$ across the anti-Mach cone, and the two other quantities are analogously defined. We separately enforced $\Delta f_{0}\left(\theta_{M}^{\prime}\right)=0$ by adding the appropriate constant to the numerical integration of $f_{0}^{\prime}$ in the intermediate region. As we varied both $f_{0}^{\prime}(\pi / 2)$ and $g_{-2}(\pi)$, the quantity $\chi$ reached a well-defined minimum. Figure 7 shows $\chi$-contours for the case $\beta=1.1$. The optimal values of $f_{0}^{\prime}(\pi / 2)$ and $g_{-2}(\pi)$, shown here by the dot within the circle, were insensitive to our choice of $\alpha$.

\subsubsection{Sample results}

With the numerical procedure in hand, we could determine the second-order flow for any desired $\beta$. Figure 8 displays 


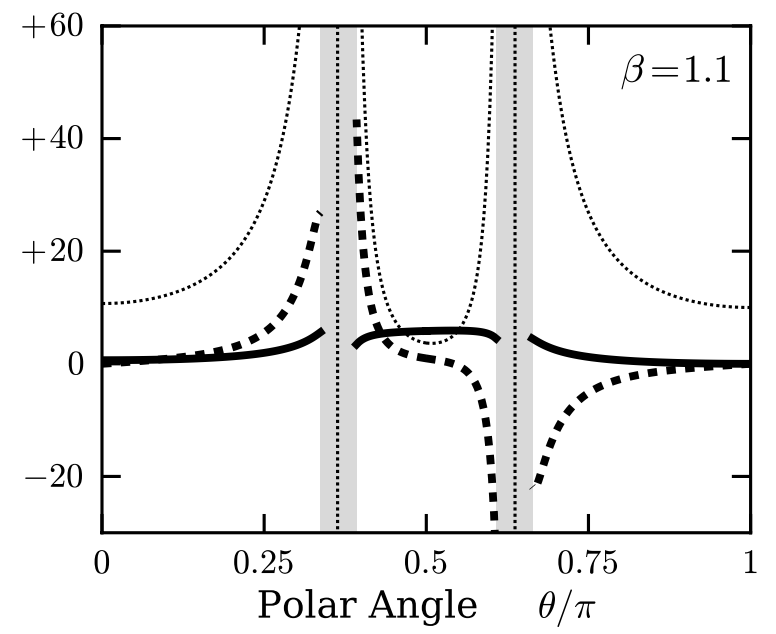

Fig. 9. Angular variation of perturbation coefficients for $\beta=1$.1. The dashed curves show $f_{0}^{\prime}$, while the dotted curves are $g_{-2}$, again using the best-fit parameters from Fig. 7. Finally, the solid curve is the integrated $f_{0}$. As in Fig. 8, all variables diverge within the shaded regions straddling the two Mach cones.

streamlines for $\beta=1.1$. Even in this modestly supersonic case, the two Mach cones depart substantially from the equatorial plane $\left(\theta_{M}=65^{\circ}\right)$, and the isodensity contours are a set of nearly vertical lines that bow inward slightly toward the mass. The shaded interiors of the wedges straddling each Mach cone represent the excluded sectors within which the divergent behavior dominates. We previously indicated the excluded region surrounding the Mach cone by the shading in Fig. 4.

Returning to Fig. 8, we notice that the streamlines in the intermediate region have the expected concavity, but are not precisely symmetric across the equatorial plane. For example, a cone with a half angle of $\theta=50^{\circ}$ cuts the outermost streamlines shown at a radius that is $1 \%$ closer downstream than upstream. Notice also how the innermost streamlines join onto the central mass. The surface of revolution they generate encloses the full $\dot{M}$, and the figure illustrates that mass accretion occurs in this order of approximation. However, we cannot obtain the detailed behavior of the flow as it joins onto the mass, since our perturbation series is only valid well outside $r=1$, the boundary indicated here as the central, dashed circle.

Figure 9 shows the angular variation of $f_{0}^{\prime}$ (dashed curve) and $g_{-2}$ (dotted curve) for this same $\beta$-value. As in Fig. 8, the shaded regions mark those sectors where both variables diverge. By design, the coefficients diverge antisymmetrically as either cone is approached. This behavior is not evident in the figure, which appears to show a symmetric divergence. In more detail, both $f_{0}^{\prime}$ and $g_{-2}$ first rise when approaching the Mach cone from downstream, then reach a peak and plunge downward (recall Fig. 4). They exhibit analogous behavior upstream of the antiMach cone.

The solid curve in Fig. 9 shows the integrated $f_{0}$. The upstream and downstream values of this coefficient match exactly at the anti-Mach cone. We forced this match by adjusting the integration constant within the intermediate region. The values of $f_{0}$ on either side of the Mach cone have a ratio of 1.6. This ratio depends both on our choices of $f^{\prime}(\pi / 2)$ and $g_{-2}(\pi / 2)$, and on the precise manner by which we excise the divergent region surrounding the Mach cone. Within our scheme, the mismatch is a weak function of the parameter $\alpha$.

As an additional example, Fig. 10 displays streamlines of the second-order flow for $\beta=2.0$. Here, both cones are even more

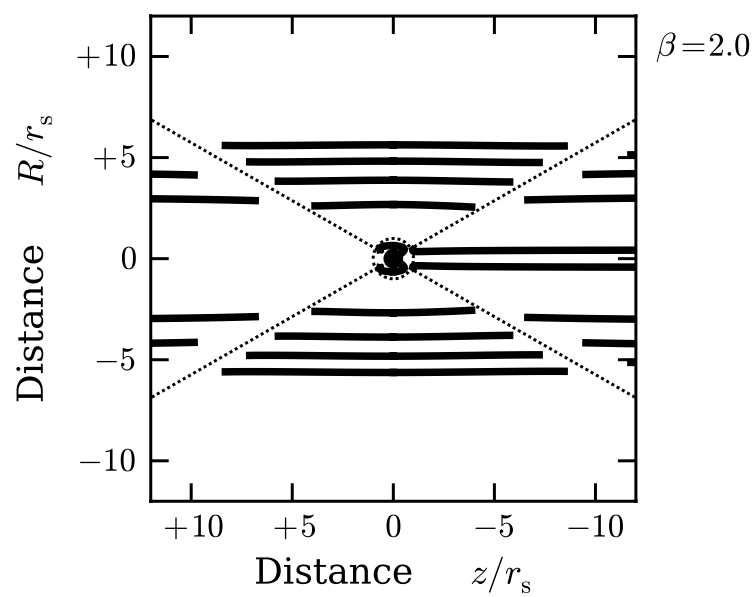

Fig. 10. Streamlines of the second-order flow for $\beta=2.0$, constructed using $f_{0}^{\prime}(\pi / 2)=0.06$ and $g_{-2}(\pi)=-0.54$. In this case, fluid elements are nearly following linear trajectories. The innermost streamlines again reach the central mass at the origin.

removed from the equatorial plane $\left(\theta_{M}=30^{\circ}\right)$, and the fluid elements are nearly following straight-line trajectories. The innermost pair shown here nevertheless still bends to reach the origin. Quantitatively, the fluid is affected significantly by gravity if it passes within the accretion radius $r_{\text {acc }}$, which varies as $\beta^{-2}$. Notice also that the figure of revolution generated by the innermost streamlines is much narrower than in Fig. 8. This narrowing reflects the fact that $\dot{M}$ decreases steeply with $\beta$ as the Mach number climbs significantly above unity.

We remind the reader that both Figs. 8 and 10 are just approximations to the actual flow. The calculated flow would become more accurate at any fixed $r \gg r_{\mathrm{s}}$ with the inclusion of higher-order terms in the perturbation series. However, the mathematical divergences at the Mach and anti-Mach cones would remain. Because of these divergences, streamlines tend to bend unphysically toward both cones. Our procedure above was designed to prevent these divergences from unduly influencing the shapes of the streamlines, at least to this order in the perturbation expansion.

\section{Friction force}

\subsection{Integration of the momentum flux}

Our reconstruction of the streamlines has a degree of arbitrariness, necessitated by the near-cone divergence in our perturbation series. We will now show that the force evaluation does not have this limitation, but is exact. Dimensionally, the total rate at which $z$-momentum is transported into a sphere surrounding the mass is

$\dot{P}=-2 \pi \int_{0}^{\pi} \rho u_{r} u_{z} r^{2} \sin \theta \mathrm{d} \theta-2 \pi \int_{0}^{\pi} \rho c_{\mathrm{s}}^{2} r^{2} \cos \theta \sin \theta \mathrm{d} \theta$.

The first right-hand term is the net advection of momentum across the surface, while the second represents the static pressure acting on the sphere. If we let our unit of force be $2 \pi \rho_{0} c_{\mathrm{s}}^{2} r_{\mathrm{s}}^{2}$, then the corresponding non-dimensional expression is

$\dot{P}=-\int_{0}^{\pi} \rho u_{r} u_{z} r^{2} \sin \theta \mathrm{d} \theta-\int_{0}^{\pi} \rho r^{2} \cos \theta \sin \theta \mathrm{d} \theta$.

The next step is to write all velocity components in terms of $\psi$ and $\rho$, and then to expand the latter variables in their respective 
perturbation series. These operations produce terms proportional to $r^{2}, r^{1}, r^{0}$, etc. We are thus motivated to write

$$
\dot{P}=\dot{P}_{2} r^{2}+\dot{P}_{1} r^{1}+\dot{P}_{0} r^{0}+\ldots
$$

Since $\dot{P}$ is also the force on the mass, it should not depend on distance. It is important, therefore, to check that both $\dot{P}_{2}$ and $\dot{P}_{1}$ vanish identically. This is indeed the case. We refer the reader to Appendix A for details of the calculation. The terms in $\dot{P}$ that are proportional to negative powers of $r$ diminish at large distance and need not be calculated explicitly. For the remainder of this section, we focus on evaluating the term $\dot{P}_{0}$.

The full expression for $\dot{P}_{0}$ may be written as

$\dot{P}_{0}=-\int_{0}^{\pi}\left(\mathcal{F}_{1}+\mathcal{F}_{2}\right) \mathrm{d} \theta$

where

$$
\begin{aligned}
\mathcal{F}_{1} \equiv & \left(1+\cos ^{2} \theta\right) \beta f_{0}^{\prime}+\left(1-\beta^{2}\right) \sin \theta \cos \theta g_{-2} \\
& +f_{1} f_{1}^{\prime}+\left(f_{1}^{\prime}\right)^{2} \cot \theta,
\end{aligned}
$$

and

$$
\begin{aligned}
\mathcal{F}_{2} \equiv & \beta^{2} \sin \theta \cos \theta g_{-1}^{2}-\beta \sin \theta \cos \theta f_{1} g_{-1} \\
& -\left(1+\cos ^{2} \theta\right) \beta f_{1}^{\prime} g_{-1} .
\end{aligned}
$$

If we use Eqs. (15) and (16) for $f_{1}$ and $g_{-1}$ in the downstream region, then we find that this contribution to the full $\dot{P}_{0}$, which we label $\dot{P}_{0}^{d}$, is

$$
\dot{P}_{0}^{d}=-\int_{0}^{\theta_{M}} \mathrm{~d} \theta\left[\left(1-\beta^{2}\right) \sin \theta \cos \theta g_{-2}+\beta\left(1+\cos ^{2} \theta\right) f_{0}^{\prime}\right] \text {. }
$$

At this point, we may utilize the condition of irrotationality, expressed as Eq. (44). Multiplying this latter equation by $(\sin \theta \cos \theta / 2)$ gives

$$
\begin{aligned}
-\left(1-\beta^{2}\right) \sin \theta \cos \theta g_{-2} & -\beta \cos ^{2} \theta f_{0}^{\prime} \\
& =\sin \theta \cos \theta\left(\frac{1}{\mathcal{D}}-\frac{2}{\sqrt{\mathcal{D}}}+\frac{1}{1+\sqrt{\mathcal{D}}}\right) .
\end{aligned}
$$

Substitution of this last relation into Eq. (57) transforms the latter into

$$
\begin{aligned}
\dot{P}_{0}^{d}= & \int_{0}^{\theta_{M}} \mathrm{~d} \theta \sin \theta \cos \theta\left(\frac{1}{1+\sqrt{\mathcal{D}}}-\frac{2}{\sqrt{\mathcal{D}}}\right) \\
& +\int_{0}^{\theta_{M}} \mathrm{~d} \theta \frac{\sin \theta \cos \theta}{\mathcal{D}}-\beta \int_{0}^{\theta_{M}} f_{0}^{\prime} \mathrm{d} \theta
\end{aligned}
$$

Of the three integrals on the right-hand side, the first may be evaluated analytically, while the second and third are divergent. After doing the first integral, we have

$\dot{P}_{0}^{d}=\frac{-1-\ln 2}{\beta^{2}}+\int_{0}^{\theta_{M}} \mathrm{~d} \theta \frac{\sin \theta \cos \theta}{\mathcal{D}}-\beta \int_{0}^{\theta_{M}} f_{0}^{\prime} \mathrm{d} \theta$.

Closely analogous reasoning for the upstream contribution, to be denoted $\dot{P}_{0}^{u}$, gives us

$\dot{P}_{0}^{u}=\frac{1+\ln 2}{\beta^{2}}+\int_{\theta_{M}^{\prime}}^{\pi} \mathrm{d} \theta \frac{\sin \theta \cos \theta}{\mathcal{D}}-\beta \int_{\theta_{M}^{\prime}}^{\pi} f_{0}^{\prime} \mathrm{d} \theta$.

For the intermediate region, we must now use Eqs. (21) and (22) for $f_{1}$ and $g_{-1}$, with $C=+1$. We find, for the contribution $\dot{P}_{0}^{i}$, an equation analogous to (57):

$\dot{P}_{0}^{i}=-\int_{\theta_{M}}^{\theta_{M}^{\prime}} \mathrm{d} \theta\left[\left(1-\beta^{2}\right) \sin \theta \cos \theta g_{-2}+\beta\left(1+\cos ^{2} \theta\right) f_{0}^{\prime}\right]$.
We again avail ourselves of irrotationality, now in the form of Eq. (46). Multiplication of this last equation by $(\sin \theta \cos \theta / 2)$ gives

$$
\begin{aligned}
-\left(1-\beta^{2}\right) \sin \theta \cos \theta g_{-2} & -\beta \cos ^{2} \theta f_{0}^{\prime} \\
& =\sin \theta \cos \theta\left(\frac{1}{\mathcal{E}}-\frac{2}{\sqrt{\mathcal{E}}}+\frac{\mathcal{E}^{1 / 2}}{\beta^{2} \sin ^{2} \theta}\right) .
\end{aligned}
$$

Thus, Eq. (60) becomes

$$
\begin{aligned}
\dot{P}_{0}^{i}= & -\beta \int_{\theta_{M}}^{\theta_{M}^{\prime}} f_{0}^{\prime} \mathrm{d} \theta \\
& +\int_{\theta_{M}}^{\theta_{M}^{\prime}} \mathrm{d} \theta \sin \theta \cos \theta\left(\frac{1}{\mathcal{E}}-\frac{2}{\mathcal{E}^{1 / 2}}+\frac{\mathcal{E}^{1 / 2}}{\beta^{2} \sin ^{2} \theta}\right) .
\end{aligned}
$$

The integrand within the second right-hand term of Eq. (61) is an odd function. Since the range of integration is symmetric about $\theta=\pi / 2$, this term vanishes, and we are left with

$$
\dot{P}_{0}^{i}=-\beta \int_{\theta_{M}}^{\theta_{M}^{\prime}} f_{0}^{\prime} \mathrm{d} \theta \text {. }
$$

Combining Eqs. (58), (59), and (62), we find

$$
\begin{aligned}
\dot{P}_{0} & =\dot{P}_{0}^{d}+\dot{P}_{0}^{u}+\dot{P}_{0}^{i} \\
& =\int_{0}^{\theta_{M}} \mathrm{~d} \theta \frac{\sin \theta \cos \theta}{\mathcal{D}}+\int_{\theta_{M}^{\prime}}^{\pi} \mathrm{d} \theta \frac{\sin \theta \cos \theta}{\mathcal{D}}-\beta \int_{0}^{\pi} f_{0}^{\prime} \mathrm{d} \theta .
\end{aligned}
$$

Within the second right-hand integral, let $v \equiv \pi-\theta$. Noting that $\pi-\theta_{M}^{\prime}=\theta_{M}$, we find

$$
\int_{\theta_{M}^{\prime}}^{\pi} \mathrm{d} \theta \frac{\sin \theta \cos \theta}{1-\beta^{2} \sin ^{2} \theta}=-\int_{0}^{\theta_{M}} \mathrm{~d} v \frac{\sin v \cos v}{1-\beta^{2} \sin ^{2} v} .
$$

Thus, the first and second right-hand integrals of Eq. (63) cancel, and we have

$$
\dot{P}_{0}=-\beta \int_{0}^{\pi} f_{0}^{\prime} \mathrm{d} \theta=\beta f_{0}(0) \text {. }
$$

Using Eq. (49), and identifying $\dot{P}_{0}$ as the friction force $F$, we arrive at our central result:

$F=\dot{M} \beta$.

Equation (65) is identical to that derived in the subsonic case, Eq. (I.58). What we have demonstrated is that a single expression for the force holds at all speeds. Given our assumption of a steady-state, laminar flow in the far field, this result is exact. Although the force involves the factor $\dot{M}$, our expression holds even for a porous, non-accreting object, such as a globular cluster traversing a galactic halo. The result also applies to a mass that is expelling its own gas, e.g., a wind-emitting star. In that case, the wind can only penetrate a limited distance before it shocks against the background flow. The dynamical friction force then acts on both the mass and its trapped wind. Our only requirement is that the background gas well outside of $r_{\mathrm{s}}$ be undisturbed.

This last stipulation is overly conservative. Mathematical convenience originally motivated our choice of $r_{\mathrm{s}}$ as the characteristic length scale. For supersonic flows, however, streamlines deviate from the background not inside $r_{\mathrm{s}}$, but instead inside $r_{\text {acc }} \sim r_{\mathrm{s}} / \beta^{2}<r_{\mathrm{s}}$. Numerical simulations show this effect (e.g., Shima et al. 1985; Ruffert 1996; Lee et al. 2013). In conclusion, it is the flow outside of $r_{\text {acc }}$ that physically determines 
Table 1. Results of Ruffert (1996).

\begin{tabular}{lccccccc}
\hline \hline$R$ & $\beta$ & $\dot{M}$ & $\dot{P}_{\text {grav }}$ & $\dot{P}_{\text {adv }}$ & $\dot{P}_{\text {tot }}$ & $\dot{P}_{\text {tot }} / \dot{M} \beta$ & $f$ \\
\hline $1.11(-1)$ & 0.6 & 2.41 & 3.19 & $-9.82(-1)$ & 2.21 & 1.53 & 1.21 \\
$2.04(-1)$ & 1.4 & $9.94(-1)$ & $1.08(+1)$ & -6.63 & 4.14 & 2.98 & 1.17 \\
$4.44(-3)$ & 3.0 & $7.86(-2)$ & 2.20 & -1.26 & $9.43(-1)$ & 4.00 & 1.23 \\
$4.00(-4)$ & 10.0 & $1.59(-3)$ & $1.78(-1)$ & $-8.91(-2)$ & $8.91(-2)$ & 5.60 & 1.33 \\
\hline
\end{tabular}

Notes. For the definitions of the quantities displayed, see Sect. 5.2 of the text. All entries are non-dimensional. Figures in parentheses are the power of ten multiplying the preceding number.

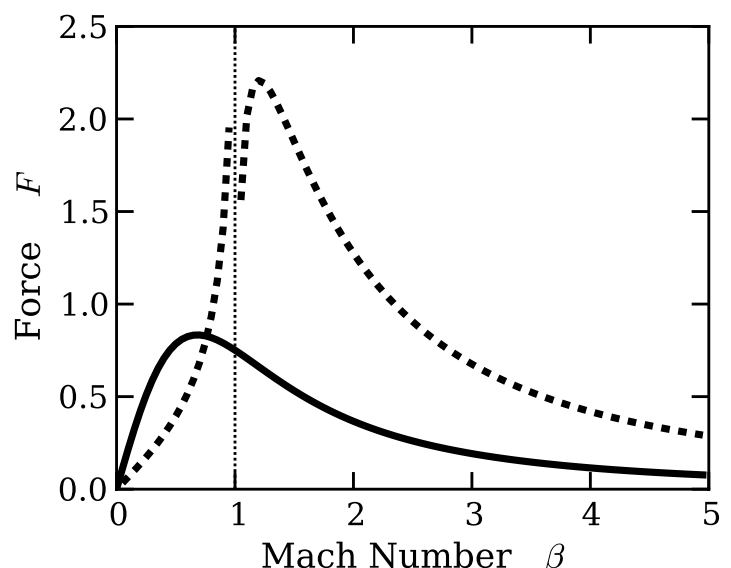

Fig. 11. The dynamical friction force $F$, shown as a function of the Mach number $\beta$. Our calculated force, taken from Eq. (66), incorporates the prescription for $\dot{M}$ from Moeckel \& Throop (2009). The dotted vertical line marks $\beta=1$. Also shown by the broken dashed curve is the force as calculated by Ostriker (1999). This force diverges at $\beta=1$.

the force, regardless of the domain of validity for our perturbation series.

For computational purposes, we may use in Eq. (65) the interpolation formula for $\dot{M}$ given in Eq. (51):

$F=\frac{2 \beta\left(\lambda^{2}+\beta^{2}\right)^{1 / 2}}{\left(1+\beta^{2}\right)^{2}}$.

We plot this relation in Fig. 11. We also show, for comparison, the friction force derived by Ostriker (1999), as given in her Eqs. (14) and (15). For $\beta>1$, her analytic formula includes the factor $\alpha_{\circ} \equiv \ln (V t / R)$, where $t$ is the time since the gravitational force is switched on. In the figure, we have set $\alpha_{\circ}=2$. Notice that Ostriker's force diverges at $\beta=1$, while ours is continuous at all Mach numbers.

\subsection{Comparison with simulations}

The numerical simulation of gas streaming past a stationary, gravitating mass has a long history. In an early work, Hunt (1971) investigated a fluid with an adiabatic index of $\gamma=5 / 3$, and with incident Mach numbers ranging from 0.6 to 2.4. A study more directly relevant to ours is that of Shima et al. (1985), whose suite of axisymmetric simulations included a $\gamma=1.1$ gas with Mach numbers again up to 2.4. The authors calculated both contributions to the friction force $F$ - the gravitational tug from the wake, determined by integration over the density around the mass, and the advection of linear momentum through the surface of this body, a force variously called the aerodynamic or hydrodynamic drag. Despite the nomenclature, they found that this second contribution is actually a forward thrust, directed oppositely to the gravitational force. Recall that external gas does not impact the mass directly, but misses it and enters from downstream, thus imparting momentum in the upstream direction.

The total force found by Shima et al. (1985) is greater than ours. These authors define a drag coefficient $c_{\mathrm{d}}$ through the relation $F=2 \pi c_{\mathrm{d}} \rho_{0} G^{2} M^{2} / V^{2}$ and an effective accretion radius $r_{\mathrm{eff}}$ through $\dot{M}=\pi r_{\text {eff }}^{2} \rho_{0} V$. It follows that

$\frac{F}{\dot{M} V}=\frac{c_{\mathrm{d}}}{2}\left(\frac{r_{\mathrm{acc}}}{r_{\mathrm{eff}}}\right)^{2}$.

Using their Table 1 and Fig. 9, we infer that, for $\beta=0.6,1.4$, and $2.4, F / \dot{M} V=1.0,2.4$, and 3.6, respectively. Note that the radius $R$ of their accreting object varied with Mach number. It was set to 0.1 times $r_{\mathrm{acc}}$, so that $R=0.2 r_{\mathrm{s}} / \beta^{2}$.

In a later study, Sánchez-Salcedo \& Brandenburg (1999) simulated the flow of an isothermal gas around a mass whose physical size $R$ excceds $r_{\text {acc }}$ by a factor of 20 or more. Under these conditions, the density enhancement in the wake is relatively mild. Sánchez-Salcedo \& Brandenburg (1999) switched on the gravity from the central mass suddenly, as in the calculation of Ostriker (1999). They found that their computed gravitational portion of the force closely matched Ostriker's analytical prediction. More recently, Kim \& Kim (2009), who adopted $\gamma=5 / 3$, showed that this contribution to the force declines when $R$ falls well below $r_{\text {acc }}$ (see their Fig. 14).

The most thorough numerical investigation of the flow pattern, mass accretion rate, and friction force for the isothermal case has been that of Ruffert (1996). In this three-dimensional simulation of a $\gamma=1.01$ gas, both the accretor size and Mach number were varied, the latter up to $\beta=10$. A general result, which corroborated and extended those of previous studies, was that the downstream region exhibited continuing instability for relatively small masses $\left(R \ll r_{\mathrm{s}}\right)$ embedded in supersonic flow. These, of course, are just the conditions of most interest for our purposes. Ruffert (1996) reported time-averaged results for both $\dot{M}$ and $F$ in such cases.

Table 1 displays the essential results found by Ruffert (1996), both for his subsonic simulations $(\beta=0.6)$ and supersonic ones. We have only taken data from the runs in which the central mass was the smallest size. Here, the radius was 0.02 times $r_{\text {acc }}$. We see that, even for $\beta=0.6, R$ is less than $r_{\mathrm{s}}$, as our own study assumes. In addition to $\beta$, other non-dimensional quantities shown in the table include: $\dot{M}$, the mass accretion rate; $\dot{P}_{\text {grav }}$, the gravitational contribution to the force; $\dot{P}_{\text {adv }}$, the advective component; and their sum $\dot{P}_{\text {tot }}$, which is also the force $F$. Notice that $\dot{P}_{\text {tot }}$ is positive, i.e., it points in the $+z$-direction in Fig. 2 . The quantity $\dot{P}_{\text {adv }}$ is negative in all cases, and smaller in magnitude than $\dot{P}_{\text {tot }}$, so that the net force indeed retards the motion of the mass relative to the background gas.

As the seventh column of the table shows, $\dot{P}_{\text {tot }}$ is not equal to $\dot{M} \beta$. Even for $\beta \sim 1$, the ratio $\dot{P}_{\text {tot }} / \dot{M} \beta$ exceeds unity, and inreases with $\beta$, as found earlier by Shima et al. (1985). If there is no error in our theoretical derivation, what could be the source of this discrepancy? 
A successful simulation must replicate the flow well in inside of $r_{\text {acc. }}$. This task becomes more demanding at higher $\beta$, since $r_{\text {acc }}$ itself varies as $\beta^{-2}$. Following how the gas joins onto the central object is especially critical. Suppose, for example, that the velocity of material just outside the gravitating mass were, for any reason, artificially low in a simulation. Then, for a given $\dot{M}$, mass conservation dictates that the density close to the object be increased. Also increased would be the value of $\dot{P}_{\text {grav }}$, which is obtained by integrating over the surrounding density. Conversely, $\dot{P}_{\text {adv }}$ would be decreased, since it is proportional to the angle-averaged incoming speed. Both factors would cause the numerically determined $\dot{P}_{\text {tot }}$ to be too large.

Only a modest lowering of the speed creates a significant rise in $\dot{P}_{\text {tot }}$. To illustrate the point, we correct for this effect by lowering the numerically calculated $\dot{P}_{\text {grav }}$ at each $\beta$-value by a factor $f$, where $f>1$. Simultaneously, we let $\dot{P}_{\text {adv }}$ be increased by the same factor. There is some value of $f$ such that $\dot{P}_{\text {tot }}$ equals $\dot{M} \beta$ :

$\dot{P}_{\text {grav }} / f+f \dot{P}_{\text {adv }}=\dot{M} \beta$.

This special $f$-value is listed in the last column of Table 1 . As $\beta$ increases by almost a factor of $20, f$ is always close to unity and varies only from 1.2 to 1.3 . Thus, relatively small errors in the two force contributions, if they are inversely related, make a big difference in $\dot{P}_{\text {tot }}$.

In an earlier paper outlining his numerical method, Ruffert (1994) stated that he softened the gravitational potential of the central mass specifically to keep the incoming velocity relatively low and thereby lengthen the computational time step (see his Sect. 2.4). Needless to say, a softened potential indeed creates such an effective deceleration. So does numerical viscosity. In the Piecewise Parabolic code that Ruffert (1994) employed, the viscosity in a region spanned by $N$ zones scales as $N^{-3}$ (Porter \& Woodward 1994, p. 319). For all his isothermal simulations, Ruffert (1996) used a relatively coarse grid surrounding the mass, which typically had five zones covering a distance outside the object equal to its radius.

Since the work of Ruffert (1996), other researchers have tackled this flow problem with improved codes. Results have evolved substantially. From Table 1, Ruffert (1996) found $\dot{M}=0.994$ for $\beta=1.4$. In comparison, Eq. (51), which is a fit from Moeckel \& Throop (2009) to their own simulations, gives $\dot{M}=0.409$ for the same $\beta$-value. A fresh determination of the force, using modern numerical techniques, would clearly be of interest.

We stress that both $\dot{M}$ and $\dot{P}_{\text {tot }}$ at each $\beta$ should not only be calculated in the vicinity of the central mass, but also through a far-field integration. Agreement of the results obtained by these two methods would, of course, strongly corroborate the accuracy of the simulation, and also be a sensitive test that the flow has indeed reached steady state ${ }^{5}$.

\subsection{Applications of the new force law}

For illustrative purposes, we first consider the deceleration of a gravitating mass traveling through a uniform gas, and subject only to the force of dynamical friction. While the mass moves, it simultaneously accretes gas. The force is the rate of change

\footnotetext{
5 Canto et al (2011) ran a simulation for $\beta=5$ and performed a surface integration of the momentum flux over a large sphere. However, they considered only the kinetic part of the flux, i.e., the first right-hand term in our Eq.(53). The second term, from thermal pressure, is of comparable magnitude.
}

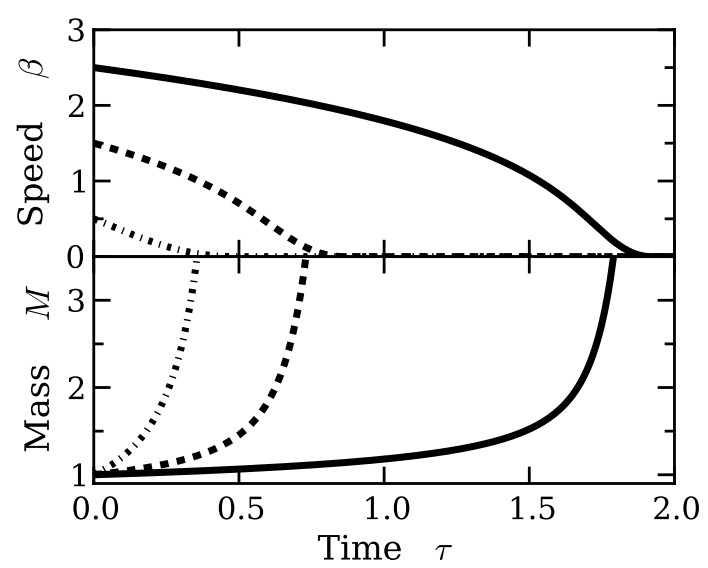

Fig. 12. Evolution of the particle's speed and mass as a function of nondimensional time $\tau$. Mass is shown relative to its initial value. The different curves represent different initial speeds: $\beta_{0}=0.5,1.5$, and 2.5 . Once the speed of an initially supersonic particle approaches $\beta \sim 1$, it quickly decelerates. Concurrently, its mass grows rapidly.

of the object's momentum, so that we have the dimensional relation

$\frac{\mathrm{d}}{\mathrm{d} t}(\dot{M} V)=-\dot{M} V$

Expansion of the derivative and integration yields

$\frac{V}{V_{0}}=\left(\frac{M}{M_{0}}\right)^{-2}$,

where $V_{0}$ and $M_{0}$ are, respectively, the object's initial speed and mass. We previously derived this result in Sect. 4 of Paper I.

Pursuing the same reasoning as before, we use $\dot{M}$ from Eq. (51) to derive the non-dimensional equation governing the velocity evolution:

$$
\left(\frac{1}{\beta}\right) \frac{\mathrm{d} \beta}{\mathrm{d} \tau}=-\frac{4\left(\lambda^{2}+\beta^{2}\right)^{1 / 2}}{\left(1+\beta^{2}\right)^{2}}\left(\frac{\beta_{0}}{\beta}\right)^{1 / 2} .
$$

Here $\beta_{0}$ is the initial speed, and $\tau \equiv t / t_{0}$ a non-dimensional time. We have used as our fiducial dimensional time

$$
t_{0} \equiv \frac{M_{0}}{2 \pi \rho_{0} c_{\mathrm{s}} r_{\mathrm{s}}^{2}} \text {. }
$$

Here the denominator is the fiducial mass accretion rate from Sect. 4.4. Thus, the quantity $t_{0}$ is a characteristic growth time for the mass when $\beta \lesssim 1$, but departs from that time in the supersonic case.

The upper and lower panels of Fig. 12 show the evolution of the velocity and mass, respectively, for various $\beta_{0}$-values. Here we obtained the mass using Eq. (67). The figure includes one subsonic case, $\beta_{0}=0.5$. Notice that, when the mass starts out at supersonic speed, it first decelerates gradually, then much more sharply as $\beta$ nears unity. Concurrently, its mass climbs rapidly, ultimately increasing at the Bondi rate appropriate for a stationary particle.

Turning to more concrete astrophysical applications, the frictional force may play an important role in the dynamical evolution of planets. In a relatively large fraction of exoplanets discovered through radial velocity studies, the normal to the orbital plane is misaligned with the stellar spin axis (Albrecht et al. 2012). Recently, Teyssandier et al. (2013) have simulated the evolution of an inclined, eccentric planet at the early epoch when 
a circumstellar disk is still present. As the planet plunges through the disk periodically, it experiences an impulsive frictional drag that gradually decreases both its orbital inclination and eccentricity, leading eventually to a coplanar, circular orbit.

In their numerical study, Teyssandier et al. (2013) found that planets with the mass of Neptune or lower can maintain their orbital inclination over the disk lifetime. On the other hand, Jovian and higher-mass planets cannot, as a result of the increased dynamical friction experienced during each disk crossing. The critical mass separating these two regimes must depend on the prescription for the frictional drag.

Teyssandier et al. (2013) used, for this drag, both that arising from direct impact with the gas and the hypersonic limit of the force derived by Ostriker (1999). In our non-dimensional units, their adopted dynamical friction force $F_{T}$ is

$F_{T}=\frac{2 I}{\beta^{2}}$,

where the factor $\mathcal{I}$ is

$\mathcal{I} \equiv \ln (H / R)$.

Here, $R$ is the radius of the planet and $H$ is the disk's semithickness, which they used in place of Ostriker's term $V t$. The ratio of our derived force $F$ to this one is

$\frac{F}{F_{T}}=\frac{\beta^{3}\left(\lambda^{2}+\beta^{2}\right)^{1 / 2}}{\left(1+\beta^{2}\right)^{2} I}$,

which approaches $\mathcal{I}^{-1}$ for $\beta \gg 1$. In their Sect. 2.4, Teyssandier et al. (2013) quote a characteristic value of $\mathcal{I} \approx 6$. Adoption of the new, diminished friction force could significantly influence the alignment of orbital planes, and thus the fraction of misaligned planets that survive the disk era.

On vastly larger scales, dynamical friction also plays a role in the assembly of supermassive black holes. Accretion onto these $10^{9} M_{\odot}$ objects powers the bright quasars detected at redshifts of $z \sim 6$, i.e., about $1 \mathrm{Gyr}$ after the Big Bang. The most popular hypothesis is that supermassive black holes arise through the merger of "seed" black holes $\left(M \sim 10^{2} M_{\odot}\right)$, which are in turn the remnants of the first stellar generation (Heger et al. 2003). The mergers occur within the dark matter haloes of coalescing galaxies. An individual black hole also accretes gas that has settled toward the center of its parent halo.

In a combined semi-analytic and numerical study, Tanaka \& Haiman (2009) showed that seed black holes can indeed form a supermassive one in the requsite time, provided they remain embedded in the halo's gas component. Mass buildup is delayed by the large recoil velocities in the coalesced product of each black hole merger. (Asymmetric gravitation radiation carries off the remaining momentum.) Promoting the merger process is dynamical friction, which can return far-flung black holes to the halo center. For their detailed numerical simulations, Tanaka \& Haiman (2009) assumed the total friction force to be

$F_{\text {tot }}=F_{\mathrm{DF}}+\dot{M} V$,

where $\dot{M}$ is the mass accretion rate of surrounding gas. They took the dynamical friction force $F_{\mathrm{DF}}$ to be the sum of that due to the collisionless sea of dark matter and that created by the gas. For the latter, Tanaka \& Haiman (2009) adopted the formula of Ostriker (1999).

According to our own study, the last term above encompasses the entire gaseous dynamical friction. That is, the dynamical friction force assumed in the simulations is too large in magnitude. A diminished $F_{\text {tot }}$ would increase the frequency of black hole ejections, as well as the time over which black holes flung outward remain on their extended orbits. In summary, the growth of supermassive black holes is delayed. As in the planetary problem, a revised calculation incorporating the corrected dynamical friction would be of interest.

\section{Discussion}

Two key assumptions underlie our derivation, and we reiterate these as a cautionary note. The first is that the geometric size of the gravitating mass be appropriately small. Specifically, we assume that $R \ll r_{\text {acc }}$. In the opposite extreme, $R \gg r_{\text {acc }}$, the main force acting on the object is not gravitational in origin, but comes from the direct impact of surrounding gas. This impact force is given dimensionally by $F_{\mathrm{imp}} \approx \pi \rho_{0} R^{2} V^{2}$. The ratio of this force to that of dynamical friction is

$\frac{F_{\mathrm{imp}}}{F} \approx \frac{1}{\dot{M} \beta^{3}}\left(\frac{R}{r_{\mathrm{acc}}}\right)^{2}$,

where $\dot{M}$ is non-dimensional. According to Eq. (51), the product $\dot{M} \beta^{3}$ approaches a constant for $\beta \gg 1$.

Our second assumption is that the gas surrounding the object be sufficiently rarefied that its self-gravity is negligible. Once the amount of mass within the radius $r_{\text {acc }}$ becomes comparable to or exceeds that of the central object, the problem becomes one of gravitational collapse. Here, accretion generally does not occur in a steady-state manner (see also Sect. 5 of Lee et al. 2013).

The present dynamical friction calculation differs sharply both in approach and result from previous efforts. Starting with Bondi \& Hoyle (1944), all other researchers have obtained expressions for the force that contain a Coulomb logarithm. This term arises from integrating over the perturbed gas, usually in the downstream wake (Bondi \& Hoyle 1944; Ostriker 1999). Dokuchaev (1964) also obtained this term, but by integrating the acoustic energy flux over a large sphere surrounding the gravitating mass. His derivation is thus closer in spirit to ours. However, we are dubious of his basic premise that the total energy loss associated with the moving mass is fully accounted for by this outgoing, acoustic disturbance. Accretion generates shocks close to the mass, and the power from radiating shocks could in principle rival the acoustic loss, for a sufficiently high velocity. On the other hand, the total inflow of linear momentum is always transported unaltered to the object itself, provided the surrounding flow is steady-state.

The strategy we adopted was to use a spherical coordinate system and expand the far-field perturbations of the stream function and density in power series in the radius $r$. We found that various coefficients in these series diverged at both the Mach and anti-Mach cones. Since the series themselves are only valid far from the gravitating mass, these divergences are purely mathematical, and result from the specific form of the perturbation series. It may be, therefore, that all divergences could be eliminated through an appropriate change of independent variables. We leave this technical issue as a topic of future study.

Turning to our specific result, we find it compelling that the friction force in the present, supersonic case is identical in form to that found in Paper I for objects moving subsonically. Our final expression is, in fact, so simple that it raises the question of whether the complex machinery we brought to bear was necessary for its derivation. As one alternative path, consider the fact that the velocity $V$ is the momentum per unit mass carried by the background gas. Multiplying $V$ by $\dot{M}$, the mass accreted per 
time, immediately yields the rate of momentum input, which is the force.

This concise derivation is intuitively appealing, but is also incorrect. While $V$ is the limiting velocity of background gas at infinite distance from the mass, the true velocity differs at any finite $r$. Similarly, the true density differs from its background value. These differences are relatively small, but they are integrated over a large surface to obtain the net inflow of momentum. As may be seen in Sect. 5.1, this inflow is $\dot{M} V$ plus several correction terms of comparable magnitude. These additional terms cancel exactly, by virtue of the fact that the flow is irrotational. A more explicit demonstration of the analogous, subsonic result is in Sect. 5.2 of Paper I.

In any event, we do agree that a simpler derivation of our result should exist, provided the far-field flow is irrotational. Equivalently, the generalized derivation must apply to barytropic fluids, i.e., those in which $P$ is a function only of $\rho$. In support of this contention, we note that Khajenabi \& Dib (2012) utilized our technique of far-field integration to show that $F=\dot{M} V$ holds for an object moving subsonically through an isentropic fluid with arbitrary adiabatic index $\gamma$. We expect that analogous reasoning will yield the same result for an isentropic fluid in the supersonic regime. At that point, the stage will be set for the new derivation, one that avoids a detailed description of the far-field flow. Whether one will still need to assume a steady-state mass accretion rate, as we have done, or be able to derive this rate from first principles, remains to be seen.

Acknowledgements. We thank Max Ruffert for a thoughtful referee report that helped to improve the clarity of the paper. Throughout this project, A.T.L. was supported by an NSF Graduate Fellowship, while S.W.S. received partial funding from NSF Grant 0908573.

\section{References}

Albrecht, S., Winn, J. N., Johnson, J. A., et al. 2012, ApJ, 757, 18

Armitage, P. J., \& Natarajan, P., 2005, ApJ, 634, 921

Bondi, H. 1952, MNRAS, 112, 195

Bondi, H., \& Hoyle, F. 1944, MNRAS, 104, 273

Cantó, J, Raga, A. C., Esquivel, A., \& Sánchez-Salcedo, F. J. 2011, MNRAS, 418, 1238

Chandrasekhar, S. 1943, ApJ, 97, 255

Chavarría, L., Mardones, D., Garay, G., et al. 2010, ApJ, 710, 583

Dodd, K. N., \& McCrea, W. J. 1952, MNRAS, 112, 205

Dokuchaev, V. P. 1964, Sov. Astron., 8, 23

El-Zant, A. A., Kim, W.-T., \& Kamionkowski, M. 2004, MNRAS, 354, 169

Heger, A., Fryer, C. L., Woosley, S. E., Langer, N., \& Hartmann, D. H. 2003, ApJ, 591, 288

Hoyle, F. \& Lyttleton, R. A. 1939, Proc. Cambridge Philos. Soc., 35, 405

Hunt, R. 1971, MNRAS, 154, 141

Khajenab, F., \& Dib, S. 2012, Ap\&SS, 340, 117

Kim, H., \& Kim, W.-T. 2009, ApJ, 703, 1278

Lee, A. T., \& Stahler, S. W. 2011, MNRAS, 416, 3177 (Paper I)

Lee, A. T., Cunningham, A. J., Mckee, C. F., \& Klein, R. I. 2013, ApJ, submitted

Matsuda, T., Inoue, M., \& Sawada, K. 1987, MNRAS, 226, 785

Moeckel. N., \& Throop, H. B. 2009, ApJ, 707, 269

Narayan, R. 2000, ApJ, 536, 663

Ostriker, E. 1999, ApJ, 513, 252

Pogorelov, N. V., Ohsugi, Y., \& Matsuda, T. 2000, MNRAS, 313, 198

Porter, D. H., \& Woodward, P. R. 1994, ApJS, 93, 209

Rephaeli, Y., \& Salpeter, E. E. 1980, ApJ, 240, 20

Ricker, P. M., \& Taam, R. E. 2008, ApJ, 672, L41

Ruderman, M. A., \& Spiegel, E. A. 1971, ApJ, 165, 1

Ruffert, M. 1994, A\&AS, 106, 505

Ruffert, M. 1996, A\&A, 311, 817

Sánchez-Salcedo, F. J., \& Brandenburg, A. 1999, ApJ, 522, L35

Shima, E., Matsuda, T., Takeda, H., \& Sawada, K. 1985, MNRAS, 217, 367

Tanaka, T., \& Haiman, Z. 2009, ApJ, 696, 1798

Teyssandier, J., Terquem, C., \& Papaloizou, J. 2013, MNRAS, 428, 658

Zel'dovich, Ya. B., \& Raizer, Yu. P. 1968, Physics of Shock Waves and HighTemperature Hydrodynamic Phenomena (New York: Academic Press), I 


\section{Appendix A: Background and first-order contributions to the momentum influx}

Here we evaluate $\dot{P}_{2}$ and $\dot{P}_{1}$, the first two coefficients on the right-hand side of Eq. (55). We will show that both coefficients are zero. The first is

$\dot{P}_{2}=-\int_{0}^{\pi}\left[\left(f_{2}^{\prime}\right)^{2} \cot \theta+2 f_{2} f_{2}^{\prime}+\cos \theta \sin \theta\right] \mathrm{d} \theta$.

Substituting $f_{2}=\beta \sin \theta / 2$, this becomes

$\dot{P}_{2}=-\left(1+\beta^{2}\right) \int_{0}^{\pi} \sin \theta \cos \theta \mathrm{d} \theta=0$.

The background flow, while it certainly carries z-momentum, makes no net contribution to the influx.

The integral expression for the second coefficient is lengthier, and we write it as

$\dot{P}_{1}=-\int_{0}^{\pi}\left(C_{1}+C_{2}\right) \mathrm{d} \theta$

where

$C_{1} \equiv 2 f_{2}^{\prime} f_{1}^{\prime} \cot \theta+2 f_{2} f_{1}^{\prime}+f_{2}^{\prime} f_{1}$,

and

$C_{2} \equiv g_{-1} \sin \theta \cos \theta-\left(f_{2}^{\prime}\right)^{2} g_{-1} \cot \theta-2 f_{2} f_{2}^{\prime} g_{-1}$.

It is convenient to split up the integral in Eq. (A.2) by region. For the downstream part, which we denote as $\dot{P}_{1}^{d}$, we use Eqs. (15) and (16) for $f_{1}$ and $g_{-1}$. We find, after algebraic simplification, that

$\dot{P}_{1}^{d}=-\int_{0}^{\theta_{M}} \mathrm{~d} \theta\left(\frac{\beta^{2} \sin \theta \cos \theta}{\mathcal{D}^{1 / 2}}+\cos \theta \sin \theta\right)$.

We now use the identity

$\frac{\mathrm{d} \mathcal{D}^{1 / 2}}{\mathrm{~d} \theta}=-\frac{\beta^{2} \sin \theta \cos \theta}{\mathcal{D}^{1 / 2}}$ to conclude that

$$
\begin{aligned}
\dot{P}_{1}^{d} & =\int_{0}^{\theta_{M}} \mathrm{~d} \mathcal{D}^{1 / 2}-\int_{0}^{\theta_{M}} \mathrm{~d}\left(\sin ^{2} \theta / 2\right) \\
& =-1-\frac{1}{2 \beta^{2}} .
\end{aligned}
$$

Similarly, the upstream part is

$$
\begin{aligned}
\dot{P}_{1}^{u} & =\int_{\theta_{M}^{\prime}}^{\pi} \mathrm{d} \mathcal{D}^{1 / 2}-\int_{\theta_{M}^{\prime}}^{\pi} \mathrm{d}\left(\sin ^{2} \theta / 2\right) \\
& =1+\frac{1}{2 \beta^{2}},
\end{aligned}
$$

so that

$\dot{P}_{1}^{d}+\dot{P}_{1}^{u}=0$.

For the intermediate piece, to be denoted $\dot{P}_{1}^{i}$, we use Eqs. (21) and (22) for $f_{1}$ and $g_{-1}$. Although we have established that $C=+1$, we will see that $\dot{P}_{1}^{i}$ is independent of this parameter, so we retain the original notation. After algebraic manipulation, we find

$\dot{P}_{1}^{i}=-\int_{\theta_{M}}^{\theta_{M}^{\prime}} \mathrm{d} \theta\left(\frac{\beta C \sin \theta \cos \theta}{\mathcal{E}^{1 / 2}}+\cos \theta \sin \theta\right)$.

Using the identity

$\frac{\mathrm{d} \mathcal{E}^{1 / 2}}{\mathrm{~d} \theta}=\frac{\beta^{2} \sin \theta \cos \theta}{\mathcal{E}^{1 / 2}}$

we infer that

$$
\begin{aligned}
\dot{P}_{1}^{i} & =-C \int_{\theta_{M}}^{\theta_{M}^{\prime}} \mathrm{d} \mathcal{E}^{1 / 2}-\int_{\theta_{M}}^{\theta_{M}^{\prime}} \mathrm{d}\left(\sin ^{2} \theta / 2\right) \\
& =0 .
\end{aligned}
$$

In summary, we have

$\dot{P}_{1}=\dot{P}_{1}^{d}+\dot{P}_{1}^{u}+\dot{P}_{1}^{i}=0$. 